\title{
Crystal deposition triggers tubule dilation that accelerates cystogenesis in polycystic kidney disease
}

\author{
Jacob A. Torres, ${ }^{1}$ Mina Rezaei, ${ }^{1}$ Caroline Broderick, ${ }^{1}$ Louis Lin, ${ }^{1}$ Xiaofang Wang, ${ }^{2}$ Bernd Hoppe, ${ }^{3}$ Benjamin D. Cowley Jr., ${ }^{4}$ \\ Vincenzo Savica, ${ }^{5}$ Vicente E. Torres, ${ }^{2}$ Saeed Khan, ${ }^{6}$ Ross P. Holmes, ${ }^{7}$ Michal Mrug, ${ }^{8,9}$ and Thomas Weimbs ${ }^{1}$
}

\begin{abstract}
"University of California Santa Barbara, Department of Molecular, Cellular, and Developmental Biology, and Neuroscience Research Institute, Santa Barbara, California, USA. ${ }^{2}$ Mayo Clinic College of Medicine, Division of Nephrology and Hypertension, Rochester, Minnesota, USA. ${ }^{3}$ University Children's Hospital Bonn, Division of Pediatric Nephrology, Bonn, Germany. ${ }^{4}$ University of Oklahoma Health Sciences Center, Department of Medicine, Section of Nephrology, Oklahoma City, Oklahoma, USA. ${ }^{5}$ University of Messina, Department of Clinical and Experimental Medicine, Messina, Italy. ${ }^{6}$ University of Florida, Department of Pathology, Gainesville, Florida, USA. 'Department of Urology and ${ }^{8}$ Department of Medicine, University of Alabama at Birmingham, Birmingham, Alabama, USA. 'Department of Veterans Affairs Medical Center, Birmingham, Alabama, USA.
\end{abstract}

\begin{abstract}
The rate of disease progression in autosomal-dominant polycystic kidney disease (ADPKD) has high intrafamilial variability, suggesting that environmental factors may play a role. We hypothesized that a prevalent form of renal insult may accelerate cystic progression and investigated tubular crystal deposition. We report that calcium oxalate (CaOx) crystal deposition led to rapid tubule dilation, activation of PKD-associated signaling pathways, and hypertrophy in tubule segments along the affected nephrons. Blocking mTOR signaling blunted this response and inhibited efficient excretion of lodged crystals. This mechanism of "flushing out" crystals by purposefully dilating renal tubules has not to our knowledge been previously recognized. Challenging PKD rat models with $\mathrm{CaOx}$ crystal deposition or inducing calcium phosphate deposition by increasing dietary phosphorus intake led to increased cystogenesis and disease progression. In a cohort of patients with ADPKD, lower levels of urinary excretion of citrate, an endogenous inhibitor of calcium crystal formation, were correlated with increased disease severity. These results suggest that PKD progression may be accelerated by commonly occurring renal crystal deposition that could be therapeutically controlled by relatively simple measures.
\end{abstract}

\section{Introduction}

Autosomal-dominant polycystic kidney disease (ADPKD) is a common, life-threatening genetic disease that causes immense human suffering and places a high economic burden on the health care system (1). Progressive renal cyst growth leads to the deterioration of renal parenchyma, and approximately $50 \%$ of patients require dialysis or kidney transplantation in adulthood (2). A vasopressin receptor antagonist was recently approved as the first treatment for ADPKD, but the utility of this drug may be limited by aquaresis, potential liver toxicity, and high cost (3). An urgent need remains for safe and effective treatment options.

Authorship note: JAT and MR are co-first authors and contributed equally to this work. Conflict of interest: JT and TW are listed inventors on a provisional patent application (62818538) by the University of California Santa Barbara (UCSB) related to discoveries reported in this paper. TW is an inventor on a patent application (15/326296) by UCSB. BC reports consulting fees from Otsuka Pharmaceuticals. MM reports grants and consulting fees from Otsuka Pharmaceuticals, Sanofi, and Chinook Therapeutics. $\mathrm{BH}$ is a consultant for Oxthera $\mathrm{AB}$, Alexion Pharmaceuticals, Dicerna Pharmaceuticals, Alnylam Pharmaceuticals, and Allena Pharmaceuticals. RH is on the Advisory Board of Chinook Therapeutics and is an inventor on 2 pending patents (15/545818 and $62 / 849564$ ) filed by Wake Forest University and UAB. VET reports grant funding from Otsuka Pharmaceuticals, Palladio Biosciences, Sanofi Genzyme, Acceleron Pharma Inc., Regulus Therapeutics, and Blueprint Medicines.

Copyright: 다 2019, American Society for Clinical Investigation.

Submitted: March 1, 2019; Accepted: July 23, 2019; Published: September 16, 2019.

Reference information: J Clin Invest. 2019;129(10):4506-4522.

https://doi.org/10.1172/JCl128503.
ADPKD is caused by mutations in either the PKD1 or PKD2 gene. Numerous functions for the respective gene products, polycystin-1 (PC1) and polycystin-2 (PC2), have been identified, but the role of polycystins in the kidney remains to be determined (4). The rate of disease progression in ADPKD has high interand intrafamilial variability, suggesting involvement of modifier genes and/or environmental factors, yet none has been clearly identified (5). If an environmental factor influences disease progression, it would be of utmost importance to identify it so that it can be controlled. Support for a role of environmental factors as determinants of disease progression in ADPKD has come from genetic experiments in mice that culminated in the widely accepted "third-hit" model of cystogenesis (6). In this model, 3 events must occur to trigger the formation of individual cysts in ADPKD: the "first hit" is a germline mutation in the PKD1 or PKD2 gene; the "second hit" is a random somatic mutation in a single tubule cell that affects the other PKD1 or PKD2 allele; and the "third hit" is an insult to the affected kidney, such as ischemic or toxic damage that triggers a repair response. Indeed, elimination of both PKD1 alleles in mature mouse kidneys alone does not lead to cyst growth for many months (7). However, subsequent renal injury in such mice - or similarly preconditioned mice - will trigger rapid cyst growth $(6,8,9)$. In human ADPKD, new cysts are thought to arise throughout life (10). Since most second hits (somatic mutations) in patients presumably occur long after kidney maturation, it is likely that subsequent third hits (damage/repair) are also required for cystogenesis in humans. However, ischemic or 
toxic kidney injuries are rare events in humans and probably do not explain the relatively constant progression of ADPKD, although it is possible that subclinical levels of such injuries may play a role. We hypothesized that a much more prevalent, and clinically relevant, mechanism may drive disease progression as a third-hit trigger and considered tubule occlusion or injury by sporadically lodged microcrystals as possibilities.

Kidneys are constantly challenged by supersaturated solutes, such as calcium oxalate $(\mathrm{CaOx})$, calcium phosphate $(\mathrm{CaP})$, uric acid, and others, which are present in the urinary filtrate and may precipitate while passing through the tubular system. Although millions of microscopic crystals may form within the kidneys daily, most are excreted safely in the urine $(11,12)$. Diet and numerous pathologies greatly influence the range of crystal burden that the kidneys experience. Up to a quarter of fresh urine samples from healthy subjects show overt crystalluria (13) without immediate harm to normal kidneys. Without innate mechanisms preventing excessive growth and retention of crystals within the luminal space, tubules would rapidly occlude and renal function would cease. Mechanisms include regulators of crystal nucleation and growth such as osteopontin, nephrocalcin, and Tamm-Horsfall protein (THP) (12). If crystals become lodged in renal tubules because of fast growth or aggregation, for example, it can lead to nephrolithiasis. The fate of such lodged microcrystals is poorly understood, but removal of these microcrystals has been proposed to occur mainly by their crossing of the epithelium into the interstitial space, leading to resorption or kidney stone formation (14). Seventy to eighty percent of all human kidney stones are composed of $\mathrm{CaOx}$, indicating that oxalate constitutes a particular challenge.

Several correlative observations support a link between ADPKD progression and renal crystal burden. Symptomatic nephrolithiasis is very common (up to $20 \%$ to $28 \%$ of cases) among patients with ADPKD (15-17), and patients with nephrolithiasis have more severe PKD than do those without (17-19). Hyperoxaluria occurs in $18 \%$ of ADPKD patients with nephrolithiasis, suggesting the presence of $\mathrm{CaOx}$ crystals in some patients (18). Patients with ADPKD also have a high incidence of clinical gout (24\%) and hyperuricemia $(>60 \%)(18,19)$, conditions that are associated with uric acid crystal formation in the kidneys. Hyperuricemia correlates with faster disease progression in ADPKD (20). In the general population, the incidence of nephrolithiasis is greater in males than in females (21). Similarly, ADPKD progresses more rapidly in males than in females (22). Likewise, male rats are naturally more prone to $\mathrm{CaOx}$ nephrolithiasis than are female rats (23). In the Han:SPRD rat model of ADPKD, male animals are affected more severely than are females (24). This dual sex bias in humans and rats may be consistent with a role of crystal deposition in accelerating ADPKD progression.

We have investigated the possible role of renal crystal deposition in affecting the rate of progression of PKD. In the process, we uncovered what we believe to be a previously unrecognized mechanism that facilitates the passage of microcrystals by tubule dilation. We report here that acute or chronic induction of $\mathrm{CaOx}$ crystal deposition in normal rats and mice led to rapid activation of MTOR and SRC/STAT3 signaling pathways, accompanied by rapid dilation of tubule diameters along the entire tubular and collecting duct system. The same signaling pathways are known to be activated in ADPKD and contribute to renal cyst growth. Approximately 7 days after acute oxalate treatment, we observed that $\mathrm{CaOx}$ crystals were cleared via the luminal space, the signaling pathways were inactivated, and tubule diameters returned to normal. Inhibition of mTOR blocked tubule dilation and led to renal accumulation of large $\mathrm{CaOx}$ crystal aggregates. These results suggest that tubule dilation is a mechanism that is activated in response to lodged microcrystals, with the apparent purpose of flushing out such crystals. Although tubule dilation in response to renal crystal burden has previously been observed in numerous studies, to our knowledge, it has not been recognized as an active protective mechanism. Furthermore, we show that chronic oxalate exposure leads to tubular $\mathrm{CaOx}$ crystal deposition in male, but not female, animals of the Han:SPRD rat model of PKD. This led to increased cystogenesis and worsening of disease progression in male, but not female, animals. Similarly, a high-phosphorus diet (HPD) caused tubular CaP crystal deposition in the Pkhd1 ${ }^{\mathrm{PCK}}$ (PCK) rat model of PKD, leading to accelerated disease progression. We show that mTOR/STAT3 signaling was similarly activated in dilated renal tubules in patients with primary hyperoxaluria type 1 (PH1), a genetic disease that leads to $\mathrm{CaOx}$ deposition and renal failure. Finally, we show that urinary citrate levels were inversely correlated with more severe disease in patients with ADPKD. Urinary citrate is known to antagonize the precipitation of $\mathrm{CaOx}$ crystals in tubule lumens. Altogether, these results suggest a model in which tubular crystal deposition normally triggers a protective mechanism leading to temporary tubule dilation. In $\mathrm{PKD}$, this mechanism acts as a third-hit trigger that causes such dilated tubules to "overshoot" to form cysts. If this mechanism affects the rate of disease progression in human ADPKD, then it may consequently be possible to treat patients with relatively simple and well-established interventions known to reduce renal crystal formation in patients with nephrolithiasis such as dietary changes, increased water intake, and treatment with citrate as a calcium chelator. Intriguingly, increased water intake $(25,26)$ and citrate treatment (27-29) have already been shown to be effective in rat models of $\mathrm{PKD}$, but the underlying mechanisms have remained elusive, and, to our knowledge, it has not previously been considered that an effective reduction of renal crystal burden may be involved.

\section{Results}

Chronic CaOx crystal deposition leads to tubule dilation and activation of PKD-associated signaling pathways. To examine whether $\mathrm{CaOx}$ crystal deposition may lead to activation of PKD-associated signaling pathways, we used a well-established mouse model of chronic $\mathrm{CaOx}$ nephrolithiasis. Animals deficient in the renal sodium-phosphate cotransporter (NPT2a) are calciuric and form renal $\mathrm{CaOx}$ crystal deposits when treated with either hydroxyproline or glyoxylate as metabolic precursors of oxalate (30). Treated animals exhibited numerous luminal $\mathrm{CaOx}$ crystal deposits visible by polarized light microscopy after 28 days, and tubule diameter dilation was apparent throughout the parenchyma (Figure 1A). Analysis with segment-specific markers revealed that proximal tubules (FITC-labeled lotus tetragonolobus lectin [LTL]), connecting tubules (calbindin 1 [CALB1]), collecting ducts (rhodaminelabeled dolichos biflorus agglutinin [DBA]), and the thick ascending 
A

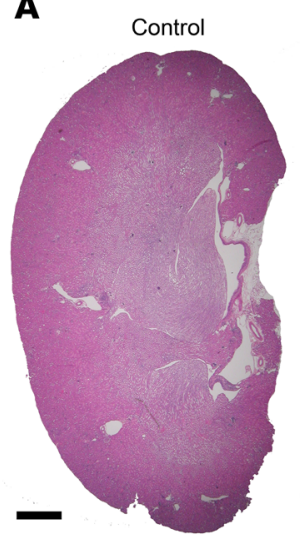

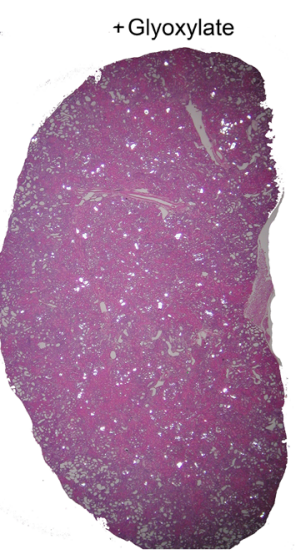
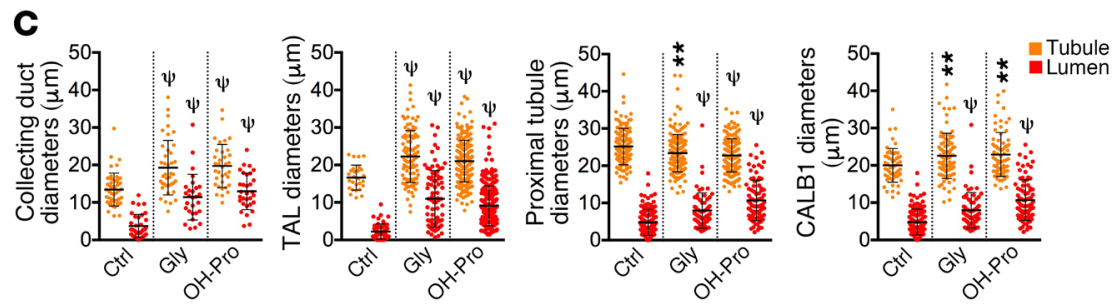

D

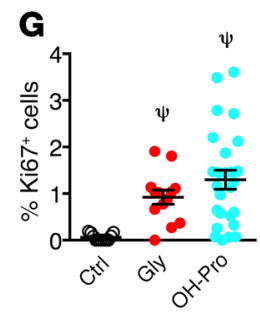

B

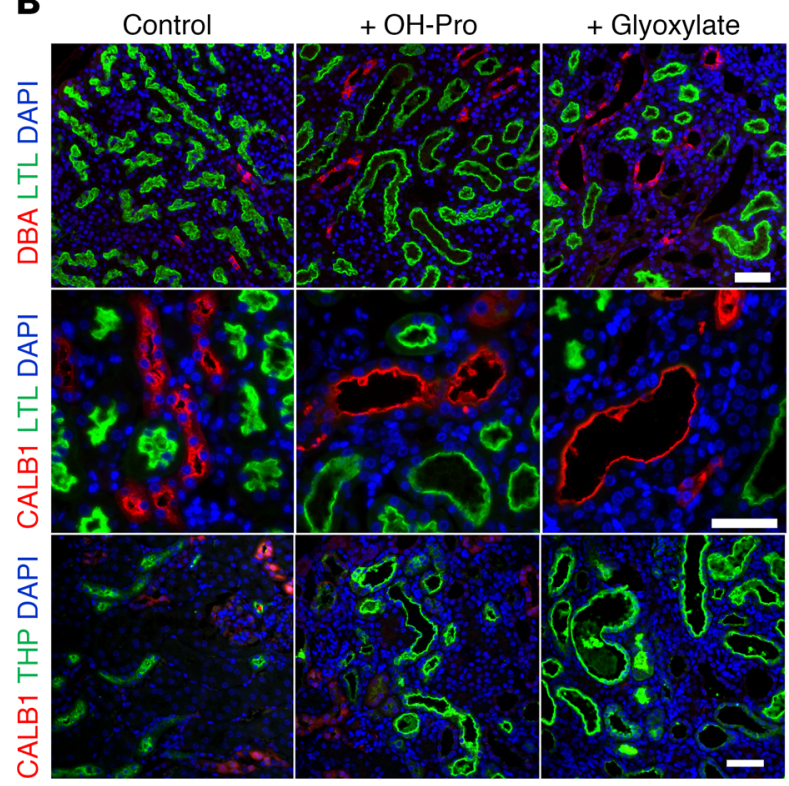

E

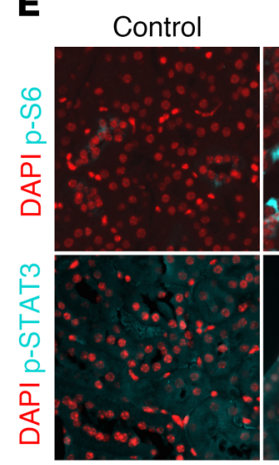

$+\mathrm{OH}-\mathrm{Pro}$

+ Glyoxylate

BPK

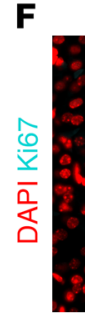

Control
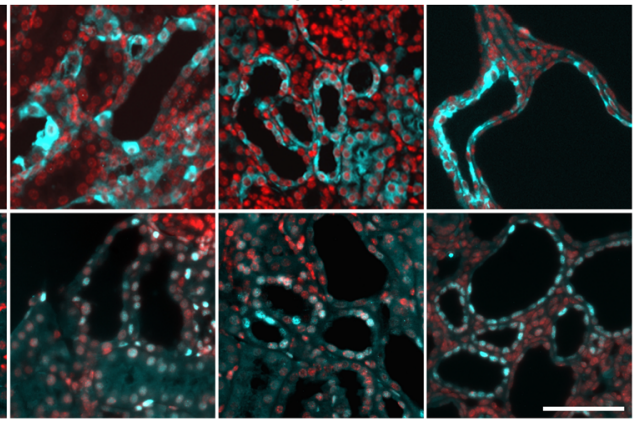

$+\mathrm{OH}-\mathrm{Pro}$

+ Glyoxylate

Figure 1. Chronic CaOx crystal deposition leads to tubule dilation and activation of PKD-associated signaling pathways. (A) H\&E-stained sections of kidneys from glyoxylate-treated $N P T 2 a^{-/-}$mice imaged by polarizing light microscopy. CaOx crystals appear as bright spots. Scale bar $=1 \mathrm{~mm}$. (B) Kidney sections from $\mathrm{NPT}_{2} \mathrm{a}^{-/-}$mice treated with glyoxylate or hydroxyproline were stained with LTL, DBA, CALB1, and THP. (C) Quantification of tubule and lumen diameters from animals in B. (D) Quantification of cell heights from animals in B. TAL, thick ascending limb of Henle. (E) Immunofluorescence microscopy to detect mTOR activity: p-S6 (Ser235/236) and p-STAT3 (Tyr705). A BPK mouse was used as a positive control. (F) Immunostaining for the cell-cycle marker Ki67. (G) Quantification of Ki67 ${ }^{+}$cells as a percentage of the total number of DAPI-stained nuclei per field analyzed. Control $n=3, n=3$ for glyoxylate, and $n=2$ for hydroxyproline. ${ }^{*} P<0.01$ and ${ }^{*} P<0.0001$, by Mann-Whitney $U$ test. Error bars represent the SD. Scale bars: $50 \mu \mathrm{m}$. Ctrl, control; Gly, glyoxylate; OH-Pro, hydroxyproline.

limb (TAL and THP) all showed dilation (Figure 1, B and C). Quantification of these segments revealed that all segments analyzed were significantly dilated (Figure 1C) and had a significant decrease in cell height (Figure 1D). Strikingly, dilated tubules were strongly positive for phosphorylated $\mathrm{S} 6$ (p-S6), a downstream effector of the mTOR signaling pathway, indicating that mTOR was activated in these cells (Figure 1E). In contrast, adjacent nondilated tubules did not show mTOR activation (Figure 1E). Likewise, dilated tubules were also strongly positive for active, nuclear tyrosine-phosphorylated STAT3 (Figure 1E). Both mTOR and STAT3 have been found to be activated in cyst-lining cells in human ADPKD and various rodent models and are drivers of renal cyst growth (31-34). For comparison, kidneys from the Bicc1 ${ }^{\text {bk }}$ (BPK) mouse model of PKD were analyzed side by side and showed a similar degree of mTOR and STAT3 activation (Figure 1E). Dilated tubules also frequently contained Ki67 $7^{+}$cells (Figure 1, F and G), indicating that chronic nephrolithiasis causes cell-cycle entry.

Rapid activation of PKD-associated signaling pathways and tubule dilation in response to acute CaOx crystal deposition. To test whether tubule dilation and activation of PKD-associated signaling pathways are slow or fast responses to $\mathrm{CaOx}$ crystal deposition, WT mice received acute treatment with a single i.p. injection of sodium oxalate $(\mathrm{NaOx})$ and were analyzed at different time points thereafter. 
A

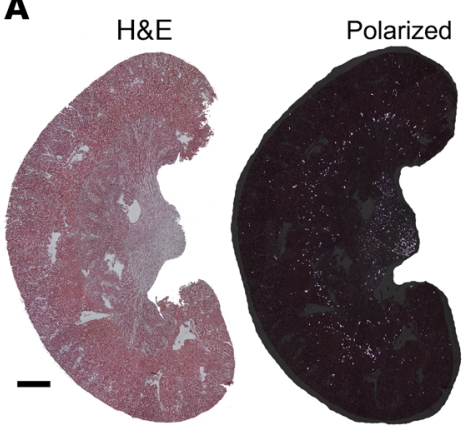

B

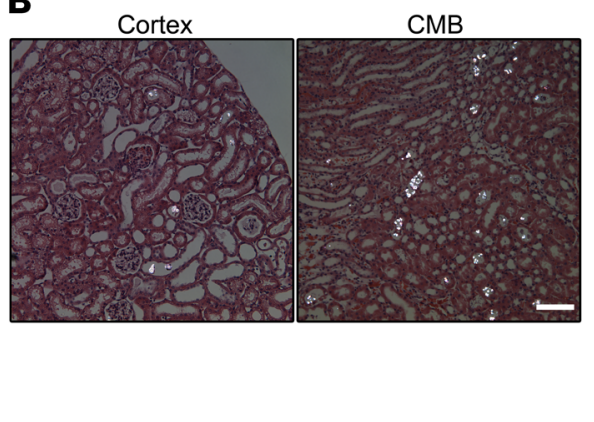

C

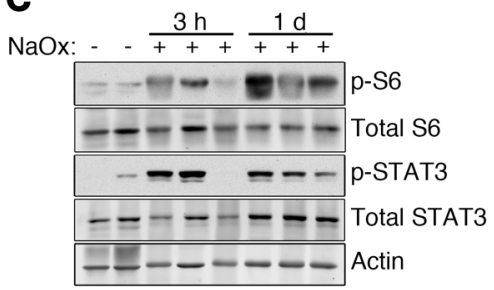

D

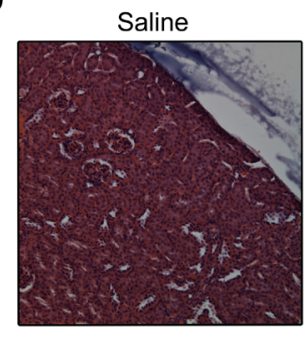

$6 \mathrm{~h}$

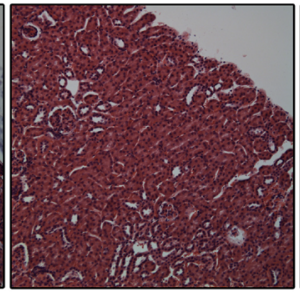

$1 d$

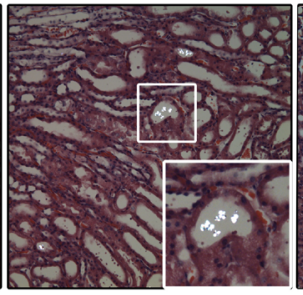

$3 d$

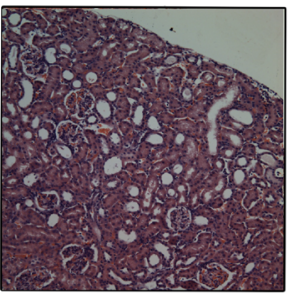

$1 \mathrm{~d}$

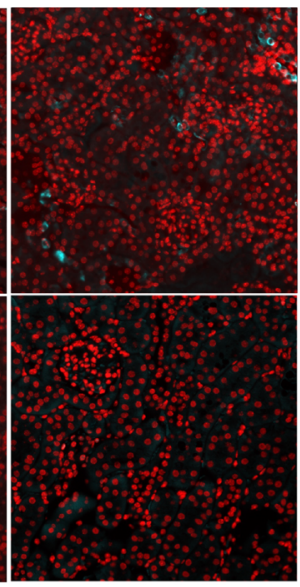

Saline

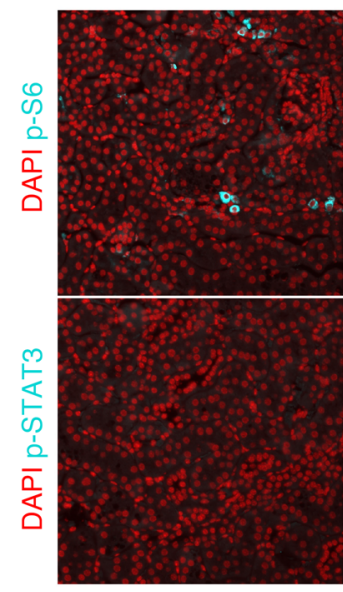

$6 \mathrm{~h}$

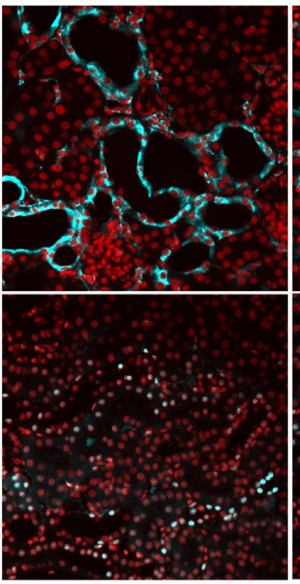

$3 d$

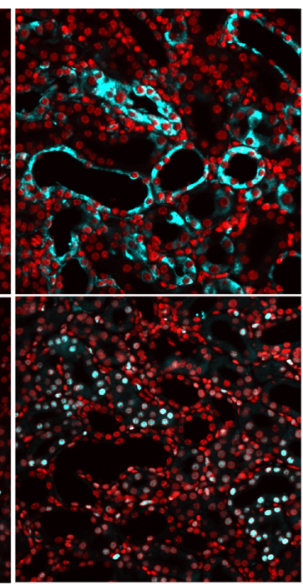

$7 d$

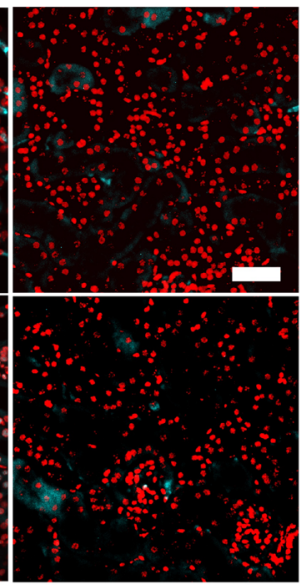

Figure 2. Acute $\mathrm{CaO}$ crystal deposition leads to rapid tubule dilation and activation of PKD-associated signaling pathways in WT C57/BL6 mice. (A) H\&E-stained kidney sections 1 day after administration of $0.7 \mathrm{mg} / \mathrm{kg} \mathrm{NaOx}$, visualized by normal and polarized light microscopy. Scale bar: $1 \mathrm{~mm}$. (B) High-magnification images from A, using polarized light. Scale bar: $100 \mu \mathrm{m}$. (C) Immunoblot of total kidney lysates for p-S6 (Ser235/236), p-STAT3 (Tyr705), and total proteins 3 hours $(n=4)$ and 1 day $(n=9)$ after $0.7 \mathrm{mg} / \mathrm{kg} \mathrm{NaOx}$ treatment. Immunoblots are representative of 2 experiments. (D) Polarized light micrographs of kidney sections from mice treated with $0.3 \mathrm{mg} / \mathrm{kg} \mathrm{NaOX}, 6$ hours $(n=4), 1$ day $(n=12), 3$ days $(n=8)$, and 7 days $(n=13)$ after treatment. Scale bar: $100 \mu \mathrm{m}$. Original magnification, x2 (inset). (E) Immunofluorescence staining of p-S6 (Ser235/236) and p-STAT3 (Tyr705) in mice treated with $0.3 \mathrm{mg} / \mathrm{kg} \mathrm{NaOx}$. Images of animals treated with $0.3 \mathrm{mg} / \mathrm{kg} \mathrm{NaOx}$ are representative of 5 experiments. Scale bar: $50 \mu \mathrm{m}$.

For unknown reasons, mice are relatively resistant to the formation of renal $\mathrm{CaOx}$ deposits $(30,35)$. A high dose of $\mathrm{NaOx}(0.7$ $\mathrm{mg} / \mathrm{kg}$ ) was initially used to induce acute, severe nephrolithiasis in the mice, which led to rapid, abundant crystal deposition that appeared to be exclusively luminal (Figure 2, A and B). This coincided with rapid activation of MTOR and STAT3 at 3 hours, peaking 1 day after treatment (Figure 2C). High-dose oxalate treatment led to substantial renal impairment and, frequently, death due to renal failure after several days.

To induce moderate, reversible crystal deposition, we next administered a low dose of $\mathrm{NaOx}(0.3 \mathrm{mg} / \mathrm{kg})$, which resulted in $\mathrm{CaOx}$ crystal deposition as early as 6 hours after injection, peaking at 24 hours, with excretion of most crystals by day 3 (Figure 2D).
Abundant $\mathrm{CaOx}$ crystals were detectable in urine from 6 hours until 3 days after injection (data not shown). We observed tubule dilation as early as 1 day after treatment, peaking at 3 days (Figure 2D). By 7 days after injection, all $\mathrm{CaOx}$ crystals were eliminated, and tubule diameters returned to normal (Figure 2D). At all the time points at which $\mathrm{CaOx}$ crystals were visible, the crystals were found nearly exclusively in tubule lumens, suggesting that the bulk of crystals were excreted in the urine via the luminal space (Figure 2D). Dilated tubules showed activation of mTOR and STAT3 as early as 1 day after $\mathrm{NaOx}$ administration, indicating that this was an early event coinciding with the process of tubule dilation (Figure 2E). Mice survived this low-dose oxalate treatment and appeared to completely recover. However, some animals failed to 
A

Saline

$6 \mathrm{~h}$

$1 d$

$3 d$
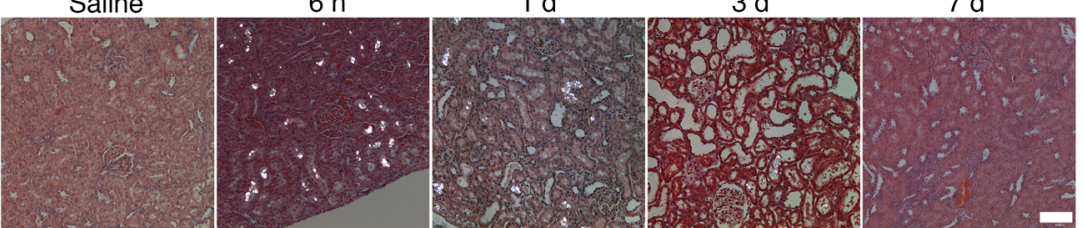

B
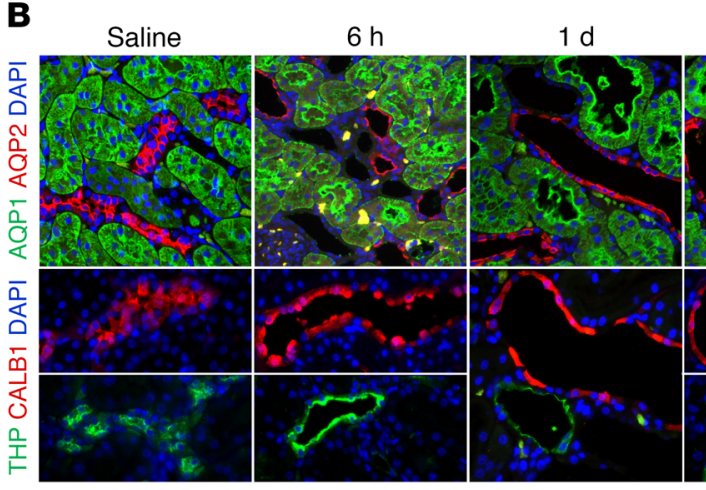

$3 d$
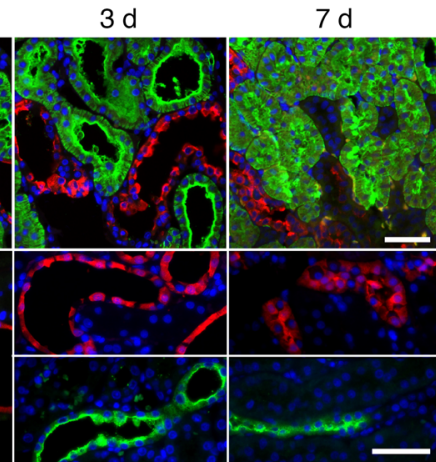

E

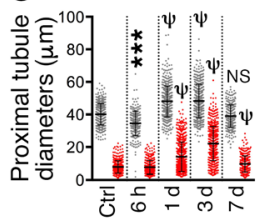

D
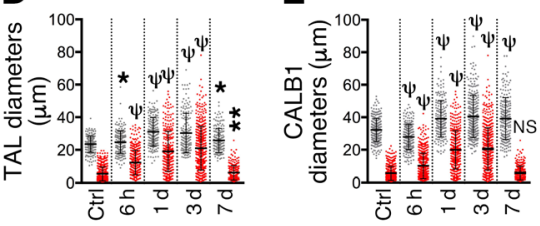

F

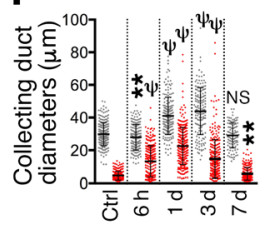

I

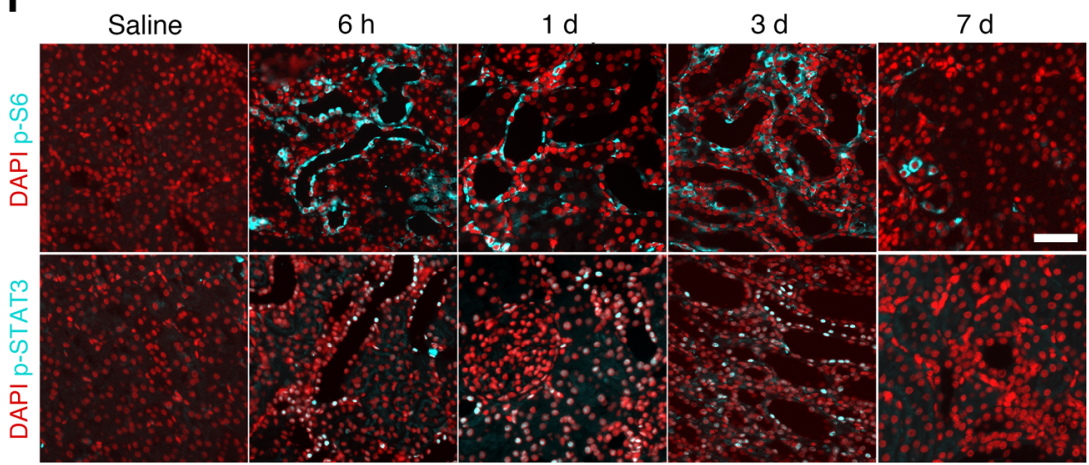

J

Saline

$6 \mathrm{~h}$

$1 \mathrm{~d}$

$3 d$

$7 d$

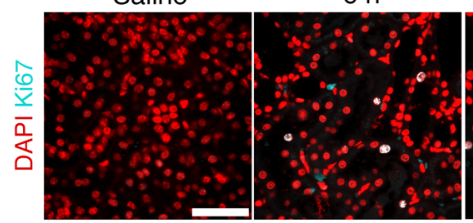

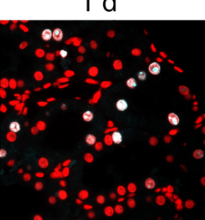

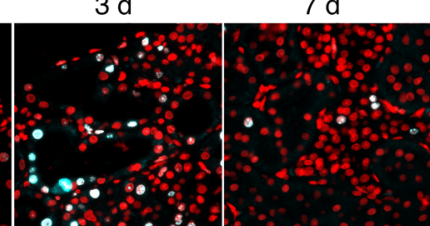

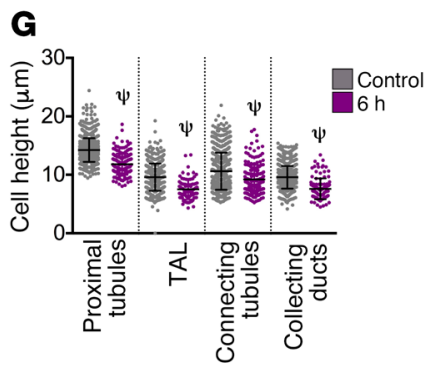

H

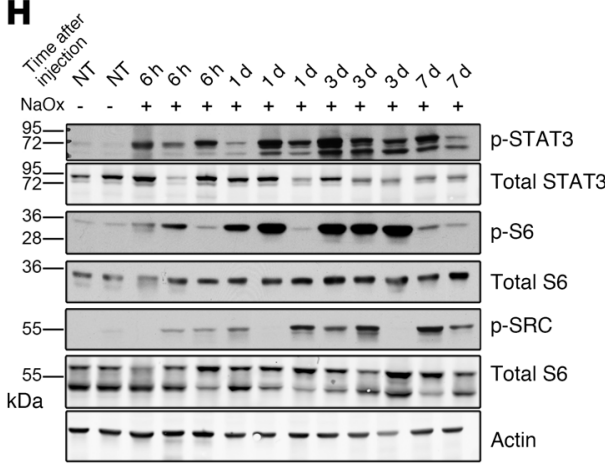

K

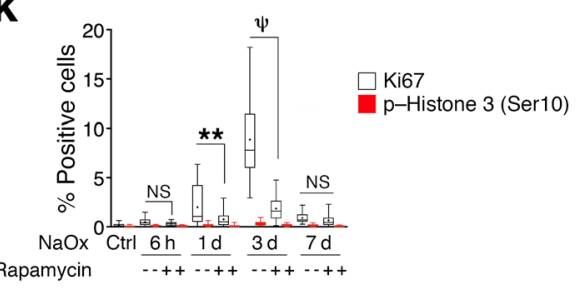

$\mathbf{L}$

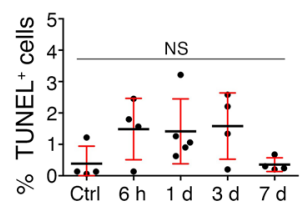

M
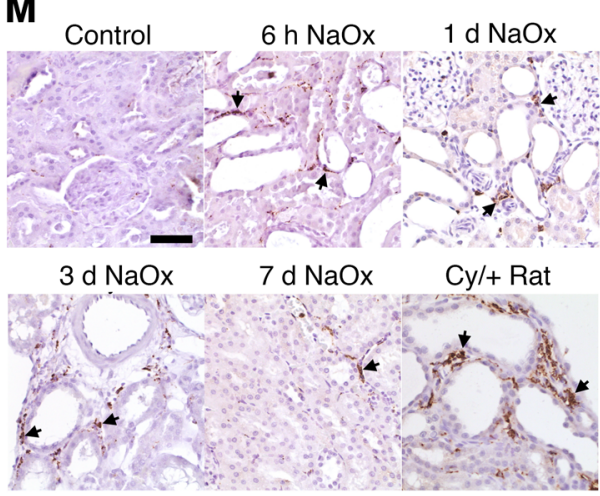
Figure 3. Acute $\mathrm{CaO}$ crystal deposition leads to rapid tubule dilation, activation of PKD-associated signaling pathways, and hypertrophy in WT rats. (A) Polarized microscopic images of H\&E-stained renal sections from rats treated with $0.7 \mathrm{mg} / \mathrm{kg} \mathrm{NaOx}, 6$ hours $(n=5), 24$ hours ( $n=5), 3$ days $(n=5)$, and 7 days $(n=3)$ after treatment. (B) Segment-specific immunostaining of collecting ducts (AQP2), proximal tubules (AQP1), connecting tubules (CALB1), and the TAL of Henle (THP) from the treated rats. Bottom panels show separate images in the same panel as necessary. Scale bars: 50 $\mu \mathrm{m}$. (C-F) Lumen and tubule diameters of the indicated tubule segments. (C) Cell heights in the indicated tubule segments 6 hours after $\mathrm{NaOx}$ challenge. (H) Immunoblot of PKD-associated signaling pathways in kidney lysates. NT, no treatment. (I) Immunofluorescence microscopic images showing p-S6 (Ser235/236) and p-STAT3 (Tyr705) at the indicated time points after $\mathrm{NaOx}$ challenge. Scale bar: $50 \mu \mathrm{m}$. (J) Ki67 immunostaining. Scale bar: $50 \mu \mathrm{m}$. (K) Kidney sections costained with Ki67 and p-histone H3 (Ser10) and quantification of positive cells as a percentage of total cells. (L) Quantification of TUNEL ${ }^{+}$cells as a percentage of total cells. (M) IHC for the macrophage marker CD68 in untreated WT rats (Control) and WT rats treated with $\mathrm{NaOx}$ at the indicated time points after $\mathrm{NaOx}$ administration. $\mathrm{Cy} /+$ rat kidney was stained for comparison. Arrows point to $\mathrm{CD68} 8^{+}$macrophages. Scale bar: $50 \mu \mathrm{m}$. Box-and-whisker plots represent $90 \%$ of the values, with the median displayed as a line in between the second and third quartiles and the mean shown with intersecting bars. All panels are representative of 3 experiments. Error bars represent the SD. ${ }^{*} P<0.05,{ }^{* *} P<0.01,{ }^{* *} P<$ 0.001 , and ${ }^{\psi} P<0.0001$, by Mann-Whitney $U$ test .

form renal CaOx crystals, which was consistent with the known resistance of mice to $\mathrm{CaOx}$ nephrolithiasis.

In order to quantify responses more accurately, we next investigated the response to moderate, reversible $\mathrm{CaOx}$ crystal deposition in a more robust experimental system. Rats are commonly used for experimental induction of $\mathrm{CaOx}$ nephrolithiasis, because they are much more prone than mice are to forming $\mathrm{CaOx}$ crystals following oxalate administration, are more resilient after crystal deposition, and recover more consistently $(36,37)$. A single injection of $\mathrm{NaOx}$ into rats again led to rapid renal $\mathrm{CaOx}$ crystal deposition within 6 hours, accompanied by fast tubule dilation (Figure 3A). After 7 days, all crystals were cleared. Analysis using segment-specific markers revealed that all examined nephron segments - namely, proximal tubules, the TAL, connecting tubules, and collecting ducts - underwent dilation (Figure 3B). Quantification of outer tubule and lumen diameters revealed that both parameters increased, peaked on day 3 , and largely returned to normal by day 7 (Figure 3, C-F). The tubule diameter increase was accompanied by a decrease in cell height (Figure 3G).

mTOR and SRC/STAT3 signaling was activated in dilated tubules 6 hours after injection and peaked on day 3 (Figure 3, $\mathrm{H}$ and I). Ki67 staining revealed that affected tubule cells entered the cell cycle as early as 6 hours after oxalate administration and peaked on day 3 (Figure 3, J and $\mathrm{K}$ ). However, very few cells stained positive for the $\mathrm{G}_{2} / \mathrm{M}$ marker p-histone $\mathrm{H} 3$ (Ser10) (38), indicating that most cells that entered the cell cycle did not proliferate but appeared to be arrested in $\mathrm{G}_{1}$ (Figure $3 \mathrm{~K}$ ), which is the hallmark of hypertrophy (39). We observed a nonsignificant increase in the number of apoptotic tubule cells (Figure 3L), indicating that this level of crystal burden does not cause extensive cell death.

We next investigated whether macrophages may be associated with tubule dilation. Macrophages are known to infiltrate cystic kidneys in a process involving monocyte chemoattractant protein 1 (Mcp1), reside in the pericystic interstitium, and promote cyst growth
$(40,41)$. Macrophages are also known to be attracted to sites of crystal deposition, which may also involve Mcp1 (12), and to promote fibrosis in $\mathrm{CaOx}$ nephrocalcinosis (42). In our study, the appearance of $\mathrm{CD} 68^{+}$macrophages coincided with tubule dilation (Figure 3M). Beginning 6 hours after oxalate treatment, increasing numbers of macrophages could be seen in the cortex and surrounding dilated tubules. After 7 days, as crystals had been excreted and most tubule diameters had returned to normal, the number of macrophages decreased but remained elevated. These results suggest that macrophages are rapidly recruited after $\mathrm{CaOx}$ challenge and may play a role in promoting tubule dilation.

Altogether, these results indicate that rats respond to renal $\mathrm{CaOx}$ crystal deposition by rapid recruitment of macrophages, with both mice and rats responding with activation of mTOR and STAT3 signaling in tubule cells, dilation of tubule diameters throughout the nephron via hypertrophy, and clearance of crystals via the luminal space for urinary excretion.

mTOR inhibition blunts tubule dilation and impairs crystal excretion. To investigate whether mTOR activation is necessary for tubule dilation in response to lodged $\mathrm{CaOx}$ crystals, rats were treated with rapamycin to inhibit mTOR, followed by acute oxalate challenge. As anticipated, we found that rapamycin treatment prevented mTOR activation (Figure 4, A and B). mTOR inhibition did not prevent the activation of SRC or STAT3 (Figure 4, A and B), indicating that these pathways are not downstream of mTOR. Rapamycin treatment greatly diminished, but did not fully suppress, cell-cycle entry as measured by Ki67 (Figure 4C and Supplemental Figure 1; supplemental material available online with this article; https://doi.org/10.1172/JCI128503DS1). Remarkably, mTOR inhibition blunted the extent of tubule dilation after oxalate challenge (Figure 4D). Eventually, all examined tubule segments were able to dilate in rapamycin-treated animals, but to a diminished degree (Figure 4E), suggesting that mTOR activity is at least partially required for tubule dilation.

mTOR inhibition led to a striking accumulation of large aggregates of $\mathrm{CaOx}$ crystals in tubules located in a distinct band at the corticomedullary boundary (CMB) (Figure 4, F-H). Immunostaining with segment-specific markers demonstrated that these clusters of $\mathrm{CaOx}$ crystals primarily accumulated in the lumens of the thin descending limb of Henle and the preceding segment of the proximal tubule (Figure $4 \mathrm{H}$ ). Crystal aggregates were still prominently observed at this border in rapamycin-treated animals 3 days after treatment (Figure 4, F and I). During this course, crystals were markedly depleted in the renal cortex in rapamycin-treated animals compared with that seen in control animals (Figure 4, F and I). These results suggest that mTOR inhibition leads to inhibition of tubule dilation, creating a "bottleneck" at the point of the nephron that naturally has the smallest lumen diameter, namely the thin descending limb of Henle. This led to accumulation of CaOx crystals at this location, which in turn prevented the efficient transport of crystals back toward the distal convoluted tubule and the collecting ducts in the renal cortex (Figure 4J). Altogether, these findings suggest that tubule dilation is a purposeful response to lodged renal crystals, that PKD-related signaling pathways are involved in this process, and that inhibition of this system leads to inefficient clearance of crystals. To our knowledge, this mechanism of crystal clearance has not been previously identified. 
A

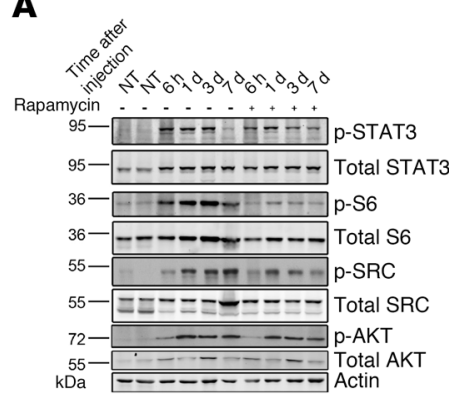

B

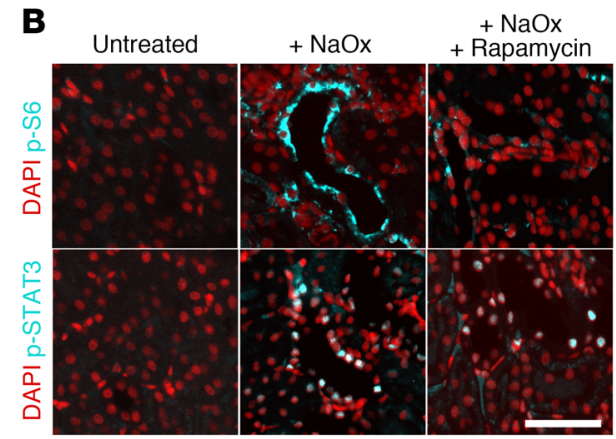

$\mathbf{C}$

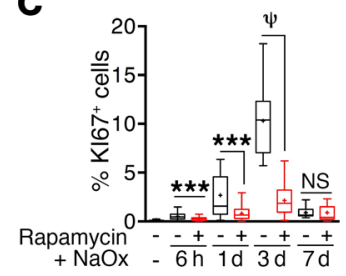

D

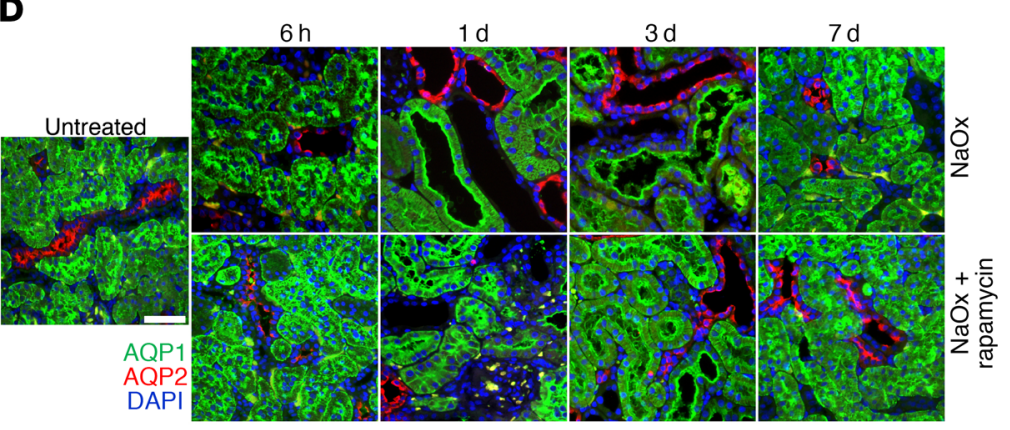

$\mathbf{F}$
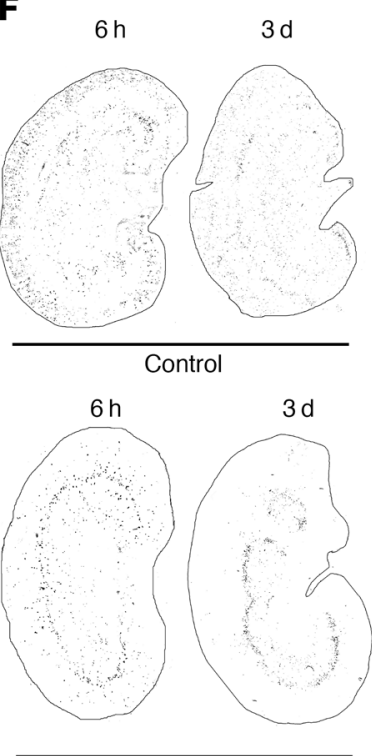

+ Rapamycin
G

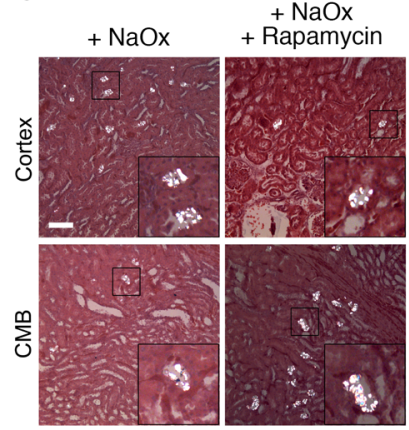

I

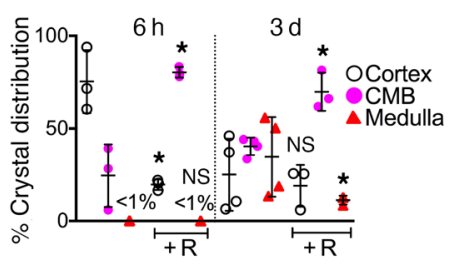

H

J
E
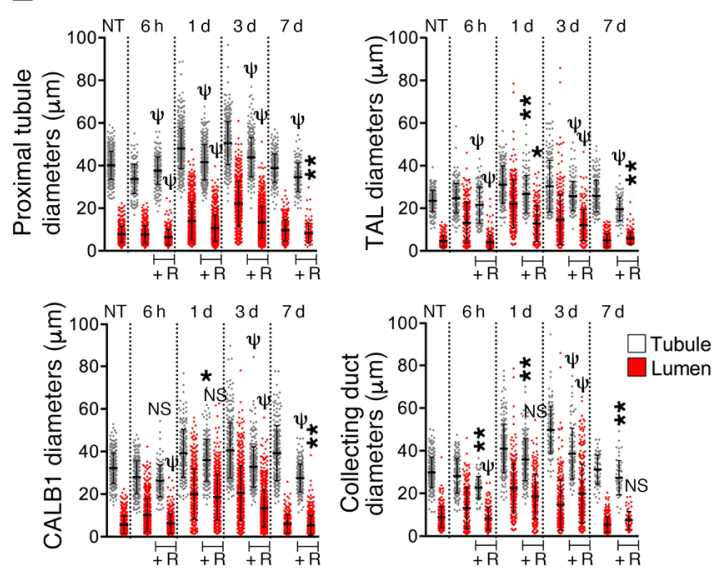
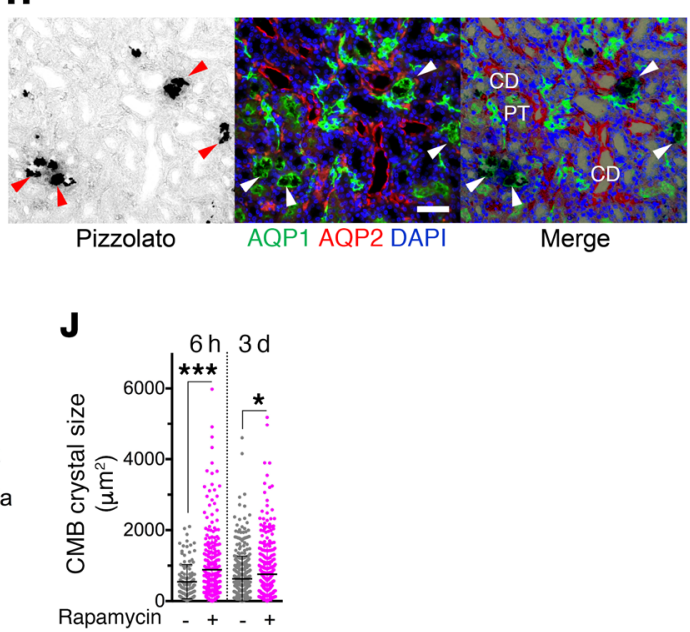

Figure 4. mTOR inhibition blunts tubule dilation and hypertrophy after NaOx challenge and disrupts efficient $\mathrm{CaO}$ crystal excretion. (A) Immunoblots of kidney lysates. (B) Immunostaining 6 hours after treatment with $\mathrm{NaOx}$, with or without rapamycin pretreatment. (C) Quantification of Ki67 ${ }^{+}$cells as a percentage of total cells. (D) Images showing immunostaining for the segment-specific markers AQP1 and AQP2. (E) Quantification of tubular and lumen diameters with rapamycin (+R). (F) Inverted polarized light micrographs of whole kidneys 6 hours or 3 days after NaOx treatment. (G) High-magnification polarized light micrographs showing oxalate crystals in renal cortex and the CMB 6 hours following NaOx treatment, with or without rapamycin pretreatment. (H) Pizzolato staining together with segment-specific markers in rapamycin-treated rats. Arrowheads point to crystals. CD, collecting duct; PT, proximal tubule. (I) Quantification of intrarenal location of deposited CaOx crystals 6 hours and 3 days after NaOx treatment, with and without rapamycin. (J) Quantification of the size distribution of $\mathrm{CaOx}$ crystals and aggregates in rats treated with $\mathrm{NaOx}$, with or without rapamycin. NaOx-treated animals after 6 hours $(n=5)$, 1 day $(n=5)$, 3 days $(n=5)$, and 7 days $(n=3)$. NaOx- plus rapamycin-treated animals after 6 hours $(n=5), 1$ day $(n=5), 3$ days $(n=5)$, and 7 days $(n=2)$. Scale bars: $50 \mu \mathrm{m}$. Original magnification, $\times 3$ (insets in G). Error bars represent the SD. All data are representative of 3 experiments for NaOx-treated animals. Box-and-whisker plots represent $90 \%$ of the values, with the median displayed as a line in between the second and third quartiles and the mean with intersecting bars. ${ }^{*} P<0.05,{ }^{* *} P<0.01,{ }^{* *} P<0.001$, and ${ }^{\psi} P<0.0001$, by Mann-Whitney $U$ test. 
A

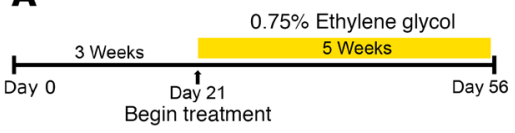

B

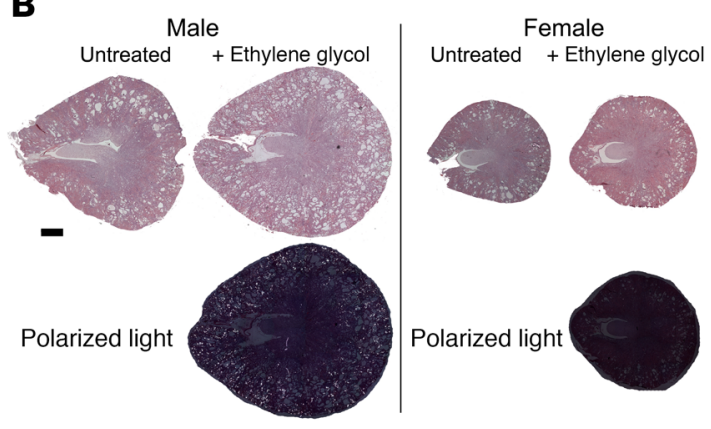

C

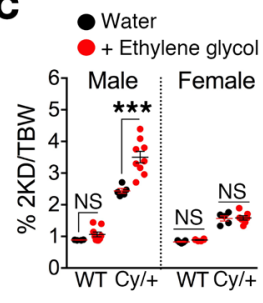

D $\bullet$ Water

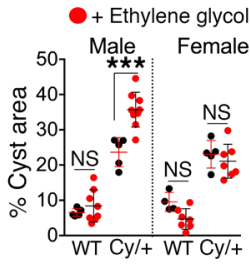

E
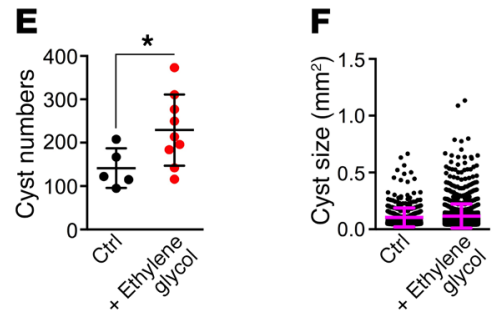

G

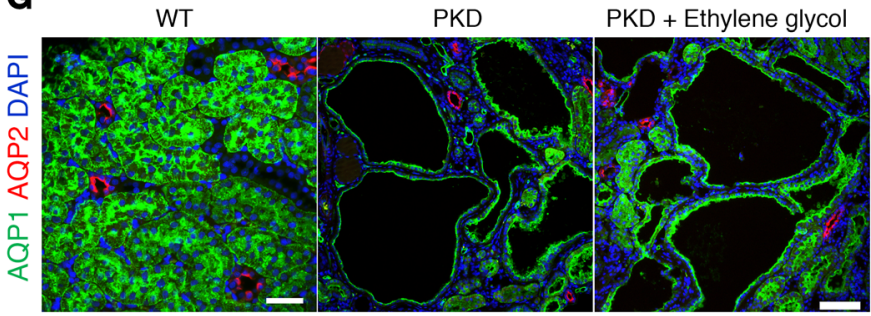

I

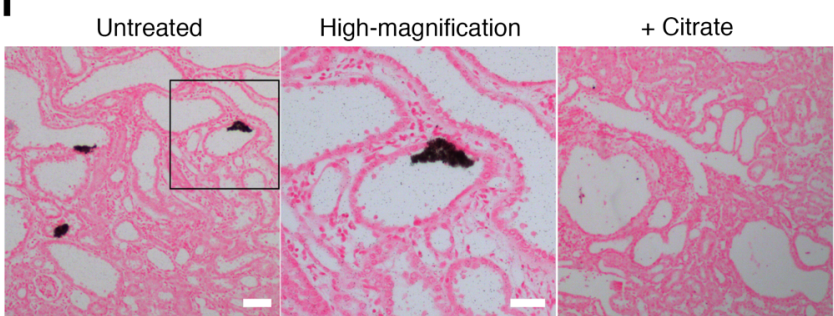

H

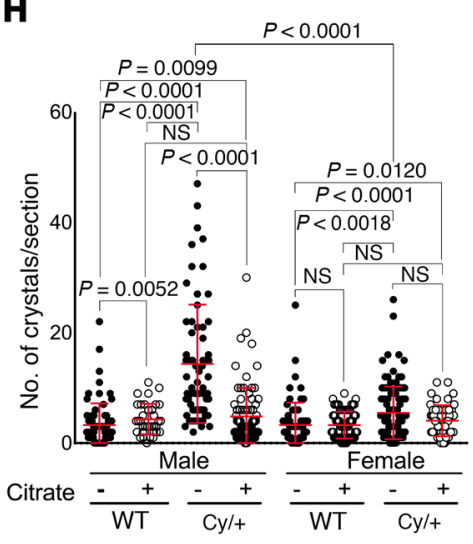

Figure 5. Chronic $\mathrm{CaOx}$ crystal deposition leads to increased cystogenesis and disease progression in the Han:SPRD rat model. (A) Timeline of treatment. Han:SPRD rats were given $0.75 \%$ ethylene glycol in their drinking water from 3 weeks of age until 8 weeks of age. (B) Bright-field and polarized light microscopic images of H\&E-stained kidney sections. Scale bar: $2 \mathrm{~mm}$. (C) Two-kidney/BW (2KD/BW) ratios. (D) Renal cystic index. (E) Cyst numbers in kidney sections per animal. (F) Cyst sizes measured by surface area in H\&E-stained sections. (G) Immunostaining for the segment-specific markers AQP1 and AQP2. Scale bars: $50 \mu \mathrm{m}$ and $100 \mu \mathrm{m}$. (H) Quantification of Pizzolato-stained tissue in I of WT and Han:SPRD rats treated with or without citrate. $P$ values in $\mathbf{H}$ were determined by Mann-Whitney $U$ test. (I) Pizzolato stained sections from untreated and citrate-treated male, Cy/+ Han:SPRD rats. Animals treated for this experiment: $n=9 \mathrm{WT}$ and $n=9 \mathrm{Cy} /+$ male rats, $6 \mathrm{WT}$ females, $n=7 \mathrm{Cy} /+$ females. Untreated animals: $n=4 \mathrm{WT}$ male rats, $n=5 \mathrm{Cy} /+$ rats, $n=5 \mathrm{WT}$ females, and $n=5 \mathrm{Cy} /+$ females. Scale bars: $100 \mu \mathrm{m}$ and $50 \mu \mathrm{m}$. Images of ethylene glycol-treated rats are representative of 4 experiments and 3 experiments for the citrate-treated rats. Error bars represent the SD. ${ }^{*} P<0.05$ and ${ }^{* * *} P<0.001$, by Mann-Whitney $U$ test.

CaOx crystal burden leads to increased disease severity in the Han:SPRD rat model of PKD. We hypothesized that renal crystal deposition may accelerate PKD progression. To investigate this possibility, we chose the well-characterized Han:SPRD (Anks $6^{\mathrm{Cy}}$ or $\mathrm{Cy} /+$ ) rat model of PKD (24), because (a) as a rat model, these animals are more susceptible to $\mathrm{CaOx}$ nephrolithiasis than are mice; (b) this is a slowly progressive model allowing for the possibility that disease progression could be accelerated; and (c) heterozygous $(\mathrm{Cy} /+)$ male animals in this model exhibit more severe disease progression than do female $\mathrm{Cy} /+$ animals, which would be consistent with the known propensity of male rats to be more susceptible to renal $\mathrm{CaOx}$ crystal deposition than female rats. We chose to investigate the early stage of PKD progression in this model (postnatal weeks $3-8$ ), as this stage represents the main period of cystogenesis during which renal function is not yet significantly affected (24).

Animals from 3 to 8 weeks of age were treated with $0.75 \%$ ethylene glycol in the drinking water (Figure 5A) for exposure to a chronic burden of $\mathrm{CaOx}$ crystals. Ethylene glycol is metabolized to oxalate, leading to hyperoxaluria, and this treatment has previously been shown to lead to $\mathrm{CaOx}$ nephrolithiasis in male, but not female, rats $(14,43,44)$. The renal effects of ethylene glycol treatment have been shown to be due to the accumulation of $\mathrm{CaOx}$ crystals, whereas other metabolic intermediates are not toxic to kidney cells at relevant concentrations (45). Numerous $\mathrm{CaOx}$ crystals were readily detectable in lumens of cysts and dilated tubules in male, but not female, $\mathrm{Cy} /+$ rats (Figure 5B). Remarkably, treated male $\mathrm{Cy} /+$ rats had more severe renal cystic 
A

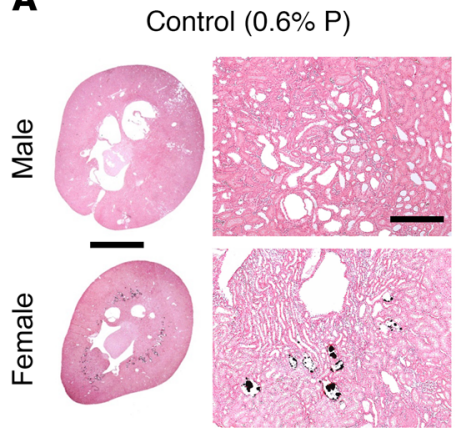

$\operatorname{HPD}(1.2 \% \mathrm{P})$

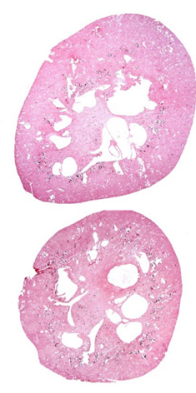

B

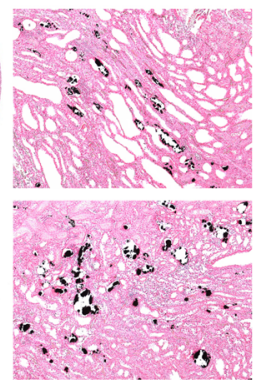

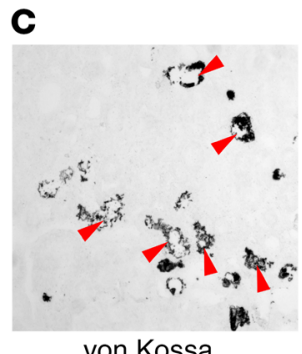

von Kossa

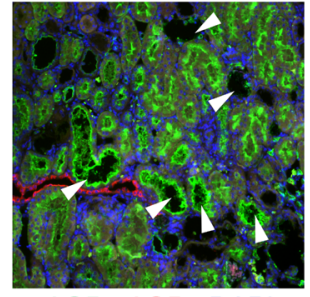

AQP1 AQP2 DAPI

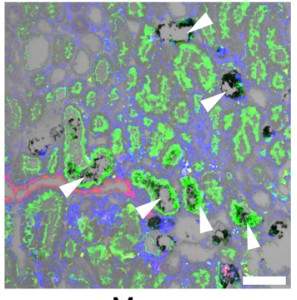

Merge

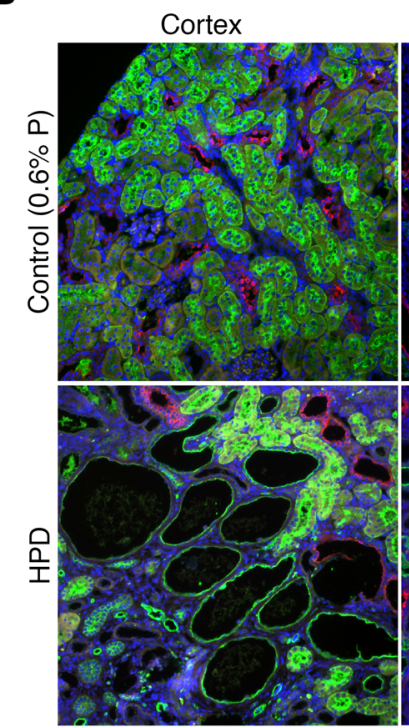

AQP1 AQP2 DAPI
D

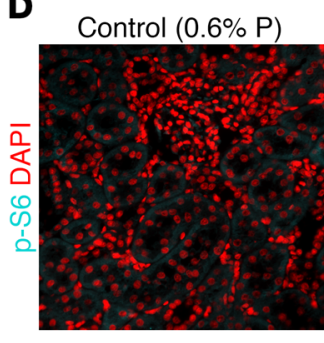

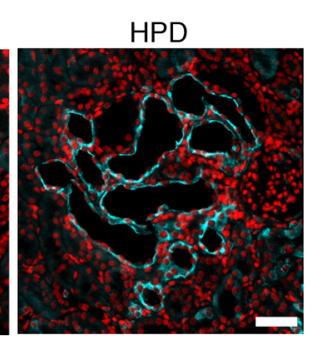

F

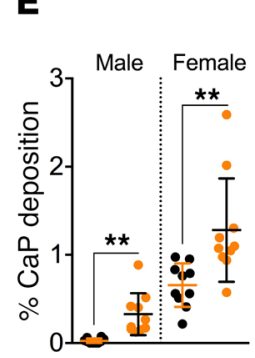

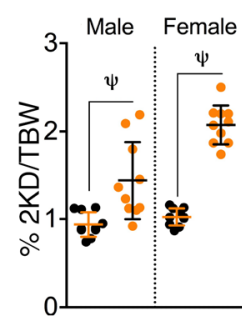

G

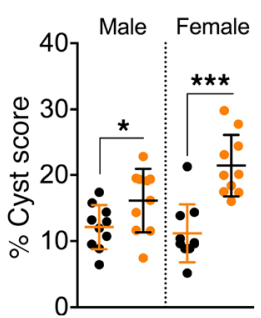

Medulla
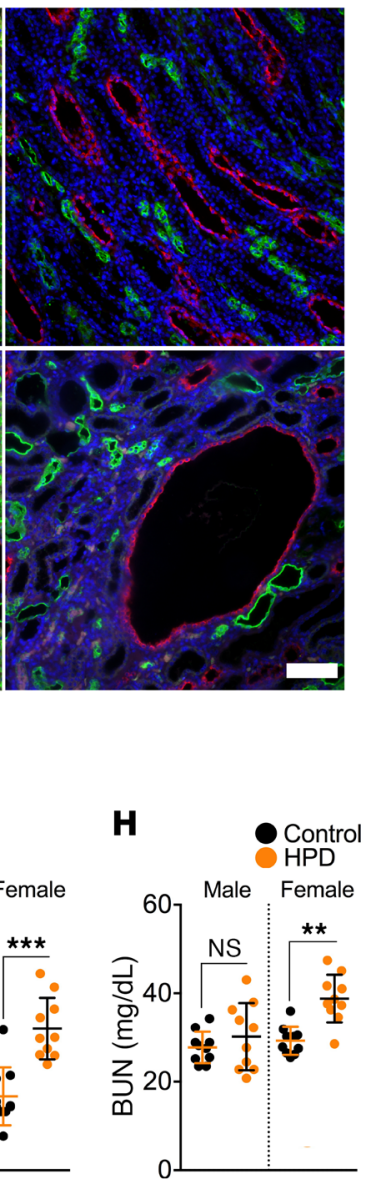

Figure 6. HPD induces renal tubular CaP deposition and accelerates disease progression in the PCK rat model. Male and female PCK rats were given a control diet containing $0.6 \% \mathrm{P}$ or a HPD containing $1.2 \% \mathrm{P}$ between 3 and 10 weeks of age. (A) Von Kossa staining showing CaP deposition (black). Scale bars: $45 \mathrm{~mm}$ and $400 \mu \mathrm{m}$. (B) Immunofluorescence microscopic images for detection of AQP1 and AQP2. Controls were cystic rats treated with $0.6 \%$ P. (C) Overlay of von Kossa stain (black) with AQP1 and AQP2 immunostaining of CaP deposits, which are seen primarily near the transition between proximal tubules and the descending limb of Henle (arrowheads). Images are from HPD-treated cystic rats. (D) Immunofluorescence staining for p-S6 (Ser235/236) in tissue from PCK rats on a HPD. (E) Quantification of CaP deposition. (F) Two-kidney/BW ratios. (G) Kidney cyst scores. (H) BUN levels for PCK rats on a HPD. $n=10$ each male and female WT and cystic rats. Error bars represent the SD. Data for E-H are from Supplemental Table 1. Scale bars: $100 \mu \mathrm{m}$ (B) and $50 \mu \mathrm{m}(\mathbf{C}$ and $\mathbf{D}) .{ }^{*} P<0.05,{ }^{* *} P<0.01,{ }^{* *} P<0.001$, and ${ }^{*} P<0.0001$, by unpaired, 2 -tailed $t$ test.

disease than did the untreated animals (Figure 5B). In contrast, disease progression was unaffected by ethylene glycol treatment in female $\mathrm{Cy} /+$ rats. The 2-kidney/BW ratio (Figure 5C) and renal cystic area (Figure 5D) were both significantly increased in ethylene glycol-treated male $\mathrm{Cy} /+$ rats versus controls. We also observed a significant increase in cyst numbers (Figure 5E), but not average cyst size (Figure $5 \mathrm{~F}$ ), suggesting that the increased disease severity was largely due to an increase in cystogenesis.

Cysts are known to arise from the proximal tubule in $\mathrm{Cy} /+$ rats (24). Immunostaining with segment-specific markers revealed that essentially all cysts were of proximal tubule origin in both ethylene glycol-treated and untreated male $\mathrm{Cy} /+$ rats (Figure 5G). This indicates that $\mathrm{CaOx}$ crystal deposition leads to increased cystogenesis only in the tubule segment that is predisposed to cyst formation in this model.

Since female $\mathrm{Cy} /+$ rats were exposed to the same ethylene glycol regimen as male $\mathrm{Cy} /+$ rats but were resistant to renal $\mathrm{CaOx}$ crystal deposition and exhibited no altered cystic disease progression, we concluded that the effect of ethylene glycol treatment on male $\mathrm{Cy} /+$ rats was not due to any chemical action of ethylene glycol or oxalate, per se, but rather to the physical interaction of $\mathrm{CaOx}$ crystals with the renal epithelium. Altogether, these results suggest that renal crystal burden can exacerbate cystogenesis, leading to overall acceleration of PKD progression.

Citrate treatment reduces CaOx crystal burden in the Han:SPRD rat model of PKD. Previously, Tanner et al. demonstrated that treatment of $\mathrm{Cy} /+$ rats with citrate leads to significant inhibition of disease progression (27-29). A mechanism for this beneficial effect could not be identified at the time, and this work never led to clinical trials. Given its ability to chelate $\mathrm{Ca}^{2+}$, citrate is a major inhibitor of $\mathrm{CaOx}$ precipitation in renal tubules and is used clinically to prevent recurring nephrolithiasis. We hypothesized that the previously observed beneficial effect of citrate therapy in the $\mathrm{Cy} /+$ rat model may involve the prevention of normally occur- 
A

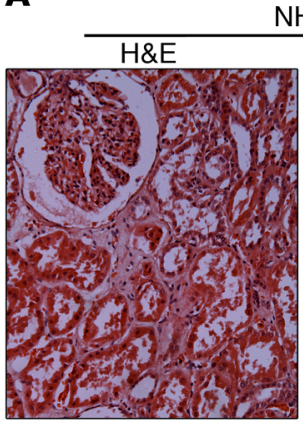

NHK

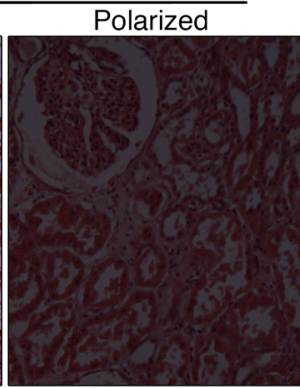

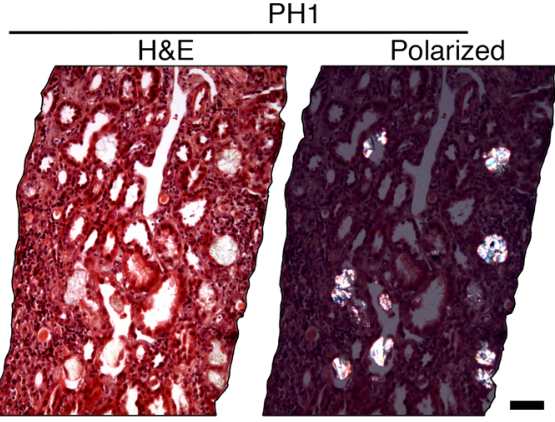

B
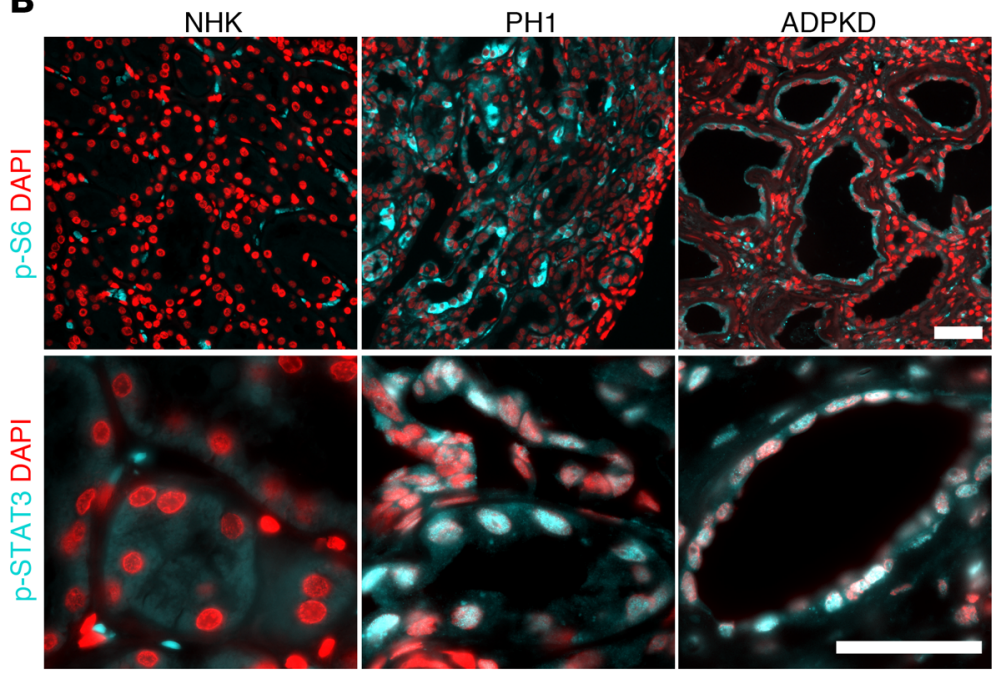

Figure 7. mTOR and STAT3 activation in kidneys from patients with PH1 and citrate excretion are inversely correlated with total kidney volume in patients with ADPKD. (A) H\&E-stained renal sections from a normal human kidney (NHK) and from a patient with PH1, visualized by bright-field and polarized light microscopy $(n=3)$. Scale bar: $50 \mu \mathrm{m}$. (B) Immunostaining for p-S6 (Ser235/236)and p-STAT3 (Tyr705) in PH1 kidneys versus NHK. Human ADPKD kidney tissue was stained side-by-side for comparison. Scale bars: $50 \mu \mathrm{m}$. (C) Urinary citrate excretion (24-hour) normalized to creatinine excretion correlated inversely with the log-transformed total renal volumes for ADPKD patients. (D) The correlation between 24-hour urine albumin excretion normalized to creatinine excretion and the log-transformed total renal volumes of ADPKD patients did not reach statistical significance. (E) Urinary citrate excretion (24-hour) normalized to creatinine and plotted against eGFR. The sample Pearson's correlation coefficients are displayed for each graph. $P$ values for C-E were determined using linear regression analysis. Hashed lines represent $95 \%$ Cls.

ring tubular Ca crystals. To test this, we treated $\mathrm{Cy} /+$ rats with citrate-supplemented drinking water as previously described (29) for 7 days and compared them with untreated animals. Very few Ca crystals were detectable in WT rats, but the male Cy/+ rats had a significantly elevated crystal burden (Figure $5 \mathrm{H}$ ). Female $\mathrm{Cy} /+$ rats showed only a marginal increase in Ca crystals compared with WT rats. Importantly, citrate treatment led to a significant reduction of Ca crystal burden in male $\mathrm{Cy} /+$ rats, reaching the baseline levels observed in WT animals (Figure 5, H and I). These results suggest that the previously observed amelioration of PKD progression by citrate therapy may be due to a reduction in renal crystal burden and consequent suppression of cystogenesis.

A high-phosphate diet leads to CaP crystal deposition and increased disease progression in the PCK rat model. Next, we investigated whether the observed effect of renal crystal deposition on PKD progression is specific to $\mathrm{CaOx}$ crystals or may also be caused by crystals of a different chemical composition, and perhaps at a different location within the tubular/collecting duct system. We investigated the PCK rat model, an orthologous model of autosomal-recessive PKD caused by a mutation in the Pkhd1 gene (46). In addition to liver cysts, PCK rats develop renal cysts predominantly of collecting duct origin that exhibit increased mTOR $(47,48)$, SRC (49), and STAT3 (50) activity.

In contrast to $\mathrm{CaOx}$ nephrolithiasis, which affects male humans and rats more severely than female humans and rats, the opposite is true for CaP nephrolithiasis $(23,51)$. Intake of phosphate varies widely among individuals and may affect the risk of $\mathrm{CaP}$ nephrolithiasis $(52,53)$. To determine whether increased dietary phosphate may affect PKD disease progression, male and female PCK rats were fed identical artificial diets except for the phosphorus content (control diet, 0.6\% P; low-phosphate diet [LPD], $0.2 \%$ P; HPD, 1.2\% P) between 3 and 10 weeks of age. A LPD caused growth retardation compared with the control diet but did not change cyst area or fibrosis (data not shown). A HPD led to a marked increase in CaP crystal deposits in tubule lumens along the corticomedullary junction, and this occurred to a larger extent in female animals than in male animals (Figure 6, A and E). The location of $\mathrm{CaP}$ crystal deposition at the corticomedullary junc- 
tion and spreading to involve the medulla are consistent with previous findings (23). Immunostaining for segment-specific markers revealed that $\mathrm{CaP}$ deposits occurred primarily in aquaporin $1^{+}$ $\left(\mathrm{AQP1}^{+}\right)$tubules at the transition between proximal tubules and the thin descending limb of Henle (Figure 6C).

Strikingly, we observed that a HPD led to a worse progression of PKD to a larger extent in female than in male animals. Twokidney/BW ratios (Figure 6F) and cyst scores (Figure 6G) were significantly increased in both male and female rats on a HPD compared with controls (see also Supplemental Table 1), and cysts were generally positive for p-S6 (Figure 6D). Increased serum blood urea nitrogen (BUN) levels in female PCK rats in the HPD group indicated impairment of renal function (Figure $6 \mathrm{H}$ ). In addition to an increase in $\mathrm{AQP}^{+}$collecting duct-derived cysts, a HPD also led to the emergence of $\mathrm{AQP1} 1^{+}$proximal tubule-derived cysts (Figure 6B). We observed no effect on liver cystic disease (Supplemental Table 1), suggesting that the effect of a HPD is kidney specific. Altogether, these results show that a diet-induced increase in tubular $\mathrm{CaP}$ precipitates leads to increased cystogenesis and PKD disease progression in this model.

Tubule dilation and activation of PKD-associated signaling pathways in human primary hyperoxaluria. To validate our findings in human disease, we examined renal tissues from patients with primary hyperoxaluria type 1 (PH1). In $\mathrm{PH} 1$, a genetic defect leads to defective glyoxylate metabolism, causing endogenous oxalate overproduction and thus hyperoxaluria, $\mathrm{CaOx}$ crystal deposition, and eventually renal failure (54). Renal biopsy specimens from patients with $\mathrm{PH} 1$ showed $\mathrm{CaOx}$ crystal deposition and tubule dilation (Figure 7A). Both mTOR and STAT3 pathways were strongly activated in $\mathrm{PH} 1$, to a degree comparable to that seen in cysts in human ADPKD (Figure 7B). This result suggests that tubular crystal deposition leads to activation of PKD-associated signaling pathways and tubule dilation in both rodents and humans.

Urinary citrate inversely correlates with disease severity in ADPKD. Since urinary citrate is a major inhibitor of $\mathrm{CaOx}$ and $\mathrm{CaP}$ crystal formation, and low urine citrate levels are a risk factor for kidney stone formation (55), we tested whether urinary citrate levels may correlate with disease severity in ADPKD. We analyzed 24-hour urine samples from a cohort of 26 ADPKD patients with varying degrees of disease progression (total kidney volume [TKV] range: 381-2787 ml; median: $1121 \mathrm{ml}$ ), but without renal functional impairment or only mild renal insufficiency based on the estimated glomerular filtration rate (eGFR) and serum creatinine levels. Oxalate excretion varied widely (range: 17-226 mg/d; median: $31 \mathrm{mg} / \mathrm{d}$ ), presumably because of fluctuating differences in dietary oxalate intake, and did not correlate with disease progression. However, urinary citrate levels correlated inversely with more advanced renal cystic disease progression as reflected by the log-transformed TKV (Figure 7C), an FDA-approved enrichment biomarker for high-risk ADPKD patients. The urinary citrate level correlated better with TKV than did the urinary albumin excretion that was previously described as an independent predictor of TKV and renal function in patients with ADPKD (Figure 7D) (56). It has previously been shown that renal clearance and urine excretion of citrate are not significantly decreased in mild renal insufficiency for other reasons unrelated to ADPKD. Consistent with this, urine citrate excretion in our ADPKD patient cohort did not significantly correlate with eGFR (Figure 7E). These results are consistent with the hypothesis that urinary citrate may be protective in patients with ADPKD by antagonizing renal calcium crystal formation.

These data complement the outcomes of our animal studies. Together, these findings are consistent with the conclusion that renal crystal deposition triggers accelerated progression of polycystic kidney disease by activation of an inherent, renoprotective mechanism that has the purpose of facilitating the excretion of tubular crystals.

\section{Discussion}

The results presented here point to a previously unrecognized renoprotective mechanism that involves dilation of renal tubules in response to lodged microcrystals with the apparent purpose of facilitating the flushing out of such crystals along the luminal space for excretion in the urine. Furthermore, our results suggest that this renoprotective mechanism acts as a third-hit trigger leading to accelerated disease progression in PKD.

Large numbers of microscopic crystals of various compositions (including $\mathrm{CaOx}, \mathrm{CaP}$, struvite, and uric acid) may form daily in human kidneys, are transported through the tubules, and are excreted safely in the urine $(11,57)$. Protective mechanisms have evolved to regulate crystal nucleation and growth and prevent functional deterioration of the kidney (12). However, the occurrence of nephrocalcinosis and nephrolithiasis indicates that these mechanisms are not always completely effective and that crystals can sporadically lodge in tubule lumens as a result of aberrantly fast growth or aggregation. It is poorly understood how sporadically lodged microcrystals are cleared from the kidneys. One observed mechanism involves some crystals that can somehow cross the epithelial barrier into the interstitial space (14), but their subsequent fate is uncertain, and this may be a slow process that occurs over a period of weeks (58). It is difficult to see how such a cumbersome and disruptive interstitial mechanism could accomplish effective clearance of more than a small amount of lodged crystals at a time. Eventually, renal crystal deposition can lead to pathological nephrocalcinosis or nephrolithiasis, including tubule occlusion and the formation of macroscopic kidney stones, which are most commonly found in the urinary space attached to the renal papilla $(59,60)$.

Even though tubule dilation is universally observed in many forms of nephrocalcinosis and nephrolithiasis in humans and animal models (61), this phenomenon has not been thought to be anything more than a secondary consequence of cell injury. Our results suggest that $\mathrm{CaOx}$ crystal deposition leads to rapid activation of mTOR and SRC/STAT3 signaling in tubule epithelial cells, accompanied by equally rapid dilation of tubule diameters. Although occasional cell damage could be observed, particularly when cells were in direct contact with a lodged crystal, the rapid dilation response occurred in most tubule segments up- or downstream of any lodged crystals, without any apparent evidence of cell damage or death. Our results suggest that segments all along an affected nephron dilate in response to lodged $\mathrm{CaOx}$ crystals, whether or not a crystal is detectable in the particular segment under observation.

Our chosen experimental conditions of oxalate challenge led to a moderate degree of initial deposition of $\mathrm{CaOx}$ crystals that could be completely resolved by the kidneys. This mild level 
of crystal burden did not lead to widespread tissue destruction or renal failure and was intended to mimic what kidneys may frequently be exposed to under nonpathological conditions. We found that $\mathrm{CaOx}$ crystals under these conditions were located almost exclusively in tubule lumens from the moment the crystals first formed until they were cleared. Interstitial crystals were very rarely observed, suggesting that the vast majority of crystals are cleared via the luminal space and excreted in the urine. The distribution of acutely induced $\mathrm{CaOx}$ crystals indicates that crystals initially formed in the proximal tubule and were then passed along the tubular system. This observation is in agreement with previous studies in rats (61) and patients with primary hyperoxaluria (62). It is intuitive that dilation along the entire length of the nephron would facilitate this mechanism of flushing out crystals and counter their accumulation. Indeed, we found that mTOR inhibition with rapamycin blunted the ability of tubules to dilate in response to $\mathrm{CaOx}$ crystals and led to the accumulation of crystal aggregates, primarily at the transition from the proximal tubule to the descending limb of Henle. These transitions were located in the CMB and appeared to represent a bottleneck for the passage of microcrystals, presumably due to the abrupt narrowing of tubule diameters. This is consistent with the previous finding of $\mathrm{CaOx}$ crystal aggregates in the same location in patients with primary hyperoxaluria (62).

We propose that tubule dilation is a purposeful mechanism that involves activation of MTOR and STAT3 signaling pathways. Several possible scenarios can be envisioned for how a $\mathrm{CaOx}$ crystal may trigger a dilation response. In a possible short-range mechanism, the direct physical contact of a crystal with the apical surface of epithelial cells may be required while the crystal passes through a tubule. This model, however, would not explain how a tubule segment distal to a lodged crystal would be able to detect its presence and dilate in response. Alternatively, in a possible long-range mechanism, a lodged crystal may activate a response both up- and downstream of its location by affecting fluid flow in the given nephron as a result of partial or complete occlusion. Another possible long-range mechanism may involve diffusible factors, such as growth factors, cytokines, or other signaling molecules, that may be secreted by injured epithelial cells in direct contact with a crystal. Primary cilia may play a role in both shortand long-range mechanisms by sensing crystals, signaling factors, and/or fluid flow. Our observation that macrophages associated rapidly with dilating tubules suggests that these infiltrating cells may also play a role in promoting tubule dilation. A role of macrophages in both nephrocalcinosis $(42,63)$ and renal cystic disease $(40,41)$ has been well established. Future work is required to investigate these possibilities.

Our results suggest that tubule dilation involves - and at least partially requires - activation of mTOR signaling. mTOR functions in 2 complexes, mTORC1 and mTORC2, which regulate numerous cellular characteristics and functions including cell size, proliferation, and the actin cytoskeleton $(64,65)$. It is possible that an mTOR-dependent cell size increase may play a role in tubule dilation. Our finding that dilation of tubule diameters came at the expense of cell height indicates that a change in cell shape is important, raising the possibility that mTOR-mediated changes in the actin cytoskeleton play a role in this process. Interestingly, $P k d 1^{-/-}$cyst-lining epithelial cells also exhibit a change in shape from cuboidal to flat (66), which suggests that PC1 may play a role in cell shape regulation. It is interesting to note that PC1 can regulate mTOR activity (32). It was also recently shown that osteoblastic cells react to stretching by PC1-dependent activation of STAT3 (67). Whether PC1 plays a direct role in the regulation of mTOR and/or STAT3 signaling during the dilation response to crystal deposition remains to be investigated.

In addition to a relatively rapid increase in tubule diameters, which peaked at 24 hours (Figure 3, C-F), we also observed a slower induction of cell-cycle entry, which peaked on day 3 after acute oxalate challenge (Figure 3K). Our results indicate that cell-cycle entry leads primarily to hypertrophy but not proliferation (Figure 3, J and $\mathrm{K})$. We hypothesize that affected cells transition from $G_{0}$ to $G_{1}$ and arrest in $G_{1}$ as they increase their cell mass. After successful completion of crystal clearance, cells then exit the cell cycle again, presumably by reverting to $G_{0}$ (Figure $3 \mathrm{~K}$ ). Therefore, ultimately, the cell numbers would remain relatively stationary, which is consistent with our finding that the response to acute $\mathrm{CaOx}$ crystal deposition led to only very minor increases in mitosis and apoptosis. It has previously been reported that acute $\mathrm{CaOx}$ crystal deposition in mice leads to necroptosis of tubule epithelial cells and an increase in $\mathrm{TUNEL}^{+}$ cells (68). However, these studies were carried out by injection with an extremely high, lethal dose of $\mathrm{NaOx}$ that was more than 300 -fold higher than that used in our study. We posit that the reason we did not observe significant cell death in our study was because we used a more physiologically relevant dose of oxalate that allowed complete crystal excretion and recovery.

mTOR activation appeared to be intimately involved in the hypertrophic response, because rapamycin treatment strongly inhibited cell-cycle entry of tubule cells in response to $\mathrm{CaOx}$ crystal deposition (Figure 4C). This is consistent with the wellestablished role of mTOR activation in hypertrophy, including the previously observed role in renal hypertrophy after unilateral nephrectomy (69). Our results, however, suggest that mTOR activation and STAT3 activation were independent of each other, because STAT3 was still activated in the presence of rapamycin (Figure $4, \mathrm{~A}$ and $\mathrm{B}$ ).

In the natural progression of $\mathrm{PKD}$, tubule dilation is the first step toward cystogenesis. Evidence from several laboratories clearly indicates that genetic inactivation of the $P k d 1$ gene or ablation of primary cilia in mice with mature kidneys is insufficient to trigger cystogenesis. However, subsequent renal insults can trigger rapid cystogenesis in such models, which has led to the now widely accepted view that a third-hit mechanism determines the rate of cystogenesis and progression in PKD (6). Experimentally, ischemia-reperfusion injury, nephrotoxic injury, and compensatory hypertrophy after unilateral nephrectomy have been shown to act as third-hit triggers in preconditioned mice. Given that such types of renal insults are rare in humans, they appear unlikely to explain the relatively steady rate of progression in human ADPKD. Our results suggest that activation of the renoprotective mechanism in response to crystal deposition described here can act as a third-hit trigger and accelerate the rate of PKD progression. Given the constant exposure of human kidneys to precipitating solutes, we consider it likely that this mechanism represents a more clinically relevant trigger that contributes to the rate of progression in ADPKD. 
In 2 rat models of PKD, we showed that conditions that lead to renal deposition of different types of crystals ( $\mathrm{CaOx}$ vs. $\mathrm{CaP}$ ) both led to increased cystogenesis and accelerated disease progression. These rat models are genetically independent of each other and exhibit different disease phenotypes. The gene coding for samcystin, a protein expressed in proximal tubule cells, is affected in the Han:SPRD (Cy/+) rat model, leading to the formation of cysts of proximal tubule origin $(24,70,71)$. In contrast, the gene coding for fibrocystin is affected in the PCK rat model (46), leading to the formation of cysts of collecting duct origin. The functions of samcystin and fibrocystin are incompletely understood. Samcystin interacts with Bicc1 (72), which is an RNA-binding protein that localizes and silences bound mRNA (73) and acts both downstream and upstream of the polycystins $(74,75)$, the proteins affected in human ADPKD. Fibrocystin, which is mutated in human ARPKD, colocalizes with the polycystins on primary cilia, physically interacts with polycystin-2 (76), and has been shown to regulate mTOR activity (77), similar to polycystin-1 (32). It is likely that most or all of the ciliopathy-associated proteins converge on the same molecular pathways that lead to renal cyst growth. PCK and Cy/+ rat models may be regarded as models for subsets of cysts that arise from distinct tubule segments. Nevertheless, despite this genetic and phenotypic distinctness between these 2 models, and despite the fact that we induced deposition of crystals of different chemical compositions, we observed an increase in cystogenesis in both cases. These results suggest that renal crystal deposition can lead to cystogenesis in a variety of tubule segments and in a variety of genetically predisposed models.

Our results indicate that the observed increase in cystogenesis was dependent on the physical presence of tubular crystals as opposed to any chemical effects of oxalate or phosphate per se. Treatment of male and female $\mathrm{Cy} /+$ rats with the same dose of ethylene glycol resulted in crystal deposition and increased cystogenesis only in the male animals. Similarly, the effect of a HPD on CaP precipitation correlated with increased cystogenesis preferentially in female rather than male PCK rats. This suggests that increased cystogenesis is due to mechanical effects of crystals such as, possibly, disruption of fluid flow or physical cell damage.

Given these results, we propose that renal crystal deposition may affect disease progression in human ADPKD. Although there is no definitive study, a link between ADPKD progression and renal crystal burden has been found in correlative studies (15-17). Generally, in these studies it is speculated that increased abnormalities in tissue architecture and/or metabolic abnormalities during ADPKD progression may increase the risk for nephrolithiasis. However, the opposite may be the case, i.e., that increased crystal burden may lead to accelerated ADPKD progression. It is also possible that ADPKD progression and nephrolithiasis positively reinforce each other, resulting in a vicious cycle.

If this conclusion is correct, then treatments that reduce renal crystal formation and deposition may slow the progression of ADPKD. Such treatments typically include dietary changes to avoid foods rich in oxalate, phosphate, and uric acid precursors; increased water intake; and supplementation with citrate as a chelator of calcium to reduce $\mathrm{CaOx}$ and $\mathrm{CaP}$ precipitation.

Remarkably, increased water intake has already been shown to be effective and slowed PKD progression in the PCK rat $(25,26)$.
The mechanism of the protective effect is unknown but was hypothesized to involve a decrease in vasopressin signaling. We hypothesize that the protective effect may instead (or in addition) be due to increased urine output and dilution of solutes, which decreases the risk of tubular crystal deposition. Increased water intake is commonly prescribed for the treatment of recurring nephrolithiasis.

Even more remarkable, citrate treatment has been shown to be highly effective in reducing cyst growth, preserving renal function, and extending lifespan in the Cy/+ rat model (27-29). The mechanism of this effect remained unexplained, and these investigators speculated that the effect may be due to urine alkalinization. In contrast, our results suggest that the observed beneficial effect of citrate was due to the action of citrate as a chelator and inhibitor of calcium crystal formation, because citrate treatment very effectively reduced the calcium crystal burden in $\mathrm{Cy} /+$ rats (Figure $5, \mathrm{H}$ and I). Citrate was found to be ineffective in the $N p h p 3^{p c y}$ mouse model of PKD $(78,79)$. However, since mice are naturally highly resistant to $\mathrm{CaOx}$ crystal precipitation, it is unlikely that tubular crystals play any role in the cystogenesis of this or any other mouse model of PKD. Therefore, citrate therapy would not be expected to be effective in such models. Furthermore, cystogenesis in most genetic mouse models of PKD is predetermined by the genetic abnormality and does not depend on any third-hit trigger. Consistent with this, no evidence for lithogenic risk factors were found in a Pkd1 mouse model (80).

In our retrospective analysis of urinary citrate excretion in patients with ADPKD, we found that lower citrate levels were significantly correlated with more advanced disease progression as reflected by the TKV (Figure 7C). Given that urinary citrate is one of the major inhibitors of calcium crystal precipitation, it is plausible that patients with high citrate excretion rates are relatively protected and exhibit a slower rate of cystic disease progression.

Altogether, our results suggest that renal tubules utilize what we believe to be a previously unrecognized mechanism, tubule dilation, to aid in the excretion of lodged luminal crystals. Activation of this mechanism also appears to inadvertently act as a third-hit trigger leading to cystogenesis in PKD. These findings suggest the intriguing possibility that disease progression in ADPKD may be favorably affected by the same therapeutic interventions that have been well established for the treatment of recurring nephrolithiasis.

\section{Methods}

Animals and treatment regimens. Animals were allowed free access to water and standard chow, weaned at 3 weeks of age, and separated by sex. All animals were monitored for signs of distress during the course of the study. Individual animals rather than animal cohorts were treated as the experimental unit for the purposes of this study. The researchers performing the experiments were not blinded to the treatment groups. Sample size was determined using prior knowledge from similar experiments performed in our laboratory involving PKD rodent models.

WT male and female C57BL/6 mice (Charles River Laboratories), 8-10 weeks of age and weighing between 20 and $30 \mathrm{~g}$, were challenged with a single i.p. injection of $0.22 \mathrm{M} \mathrm{NaOx}$ in $0.9 \%$ saline, sterile filtered and administered at $0.3 \mathrm{mg} / \mathrm{kg}$ (low dose), and euthanized at 6 hours $(n=4), 1$ day $(n=12), 3$ days $(n=8)$, and 7 days $(n=10)$; or at $0.7 \mathrm{mg} / \mathrm{kg}$ (high dose), and animals were euthanized at 3 hours $(n=$ 
4) and 1 day $(n=9)$ to induce acute $\mathrm{CaOx}$ crystal deposition. Control animals included males $(n=6)$ and females $(n=5)$ treated with saline. Low-dose trials were conducted in 5 separate experiments, and highdose trials were conducted in 2 separate experiments. Animals that did not have $\mathrm{CaOx}$ crystal deposition were not included in the analyses of crystal effects.

$\mathrm{NPT} 2 a^{-/-}$mice from a colony at the University of Florida were previously described as a model of chronic $\mathrm{CaOx}$ nephrolithiasis (36). $N P T 2 a^{-/}$mice, 4-6 months of age and weighing 18-30 g, were given $1.5 \%$ glyoxylate $(n=3)$ or $5 \%$ hydroxy proline $(n=2)$ mixed with chow for 28 days to induce hyperoxaluria. By day 7 of the treatment, $\mathrm{NPT} 2 a^{-/-}$mice became hyperoxaluric and remained so throughout the experimental period (35).

Eight-week-old Sprague Dawley rats weighing between 200 and $300 \mathrm{~g}$ (Charles Rivers Laboratories) were challenged with a single i.p. injection of $0.22 \mathrm{M} \mathrm{NaOx}$ in $0.9 \%$ saline, sterile filtered and administered at $0.7 \mathrm{mg} / \mathrm{kg}$ and were analyzed after 6 hours $(n=5), 1$ day $(n=5), 3$ days $(n=5)$, or 7 days $(n=3)$. Six-hour, 1-day, and 3-day treatments were conducted in 3 experiments and the 7-day treatment in 1 experiment. For mTOR inhibition, rats were treated with $2 \mathrm{mg} / \mathrm{kg}$ rapamycin (LC Laboratories) dissolved in DMSO 18 hours prior to, then 24 and 72 hours after, oxalate challenge. The control animals received DMSO only. Rapamycin-treated animals were treated with oxalate as described above and euthanized 6 hours $(n=5), 1$ day $(n=5), 3$ days $(n=5)$, or 7 days $(n=2)$ later. Six-hour, 1-day, and 3-day treatments were conducted in 3 experiments and the 7-day treatment in 1 experiment. Five age-matched, untreated control animals were used for this experiment. Animals that did not have crystal deposition were not included in the histological analyses.

Han:SPRD rats were obtained from the University of Oklahoma Health and Sciences Center and a colony established at the University of California Santa Barbara. The animals were genotyped as previously described (70). Ethylene glycol was administered to male and female Han:SPRD, Cy/+, and control rats to induce chronic CaOx crystal deposition as previously described (81). Ethylene glycol (0.75\%) (MilliporeSigma) in purified water was administered to animals ad libitum from 3 to 8 weeks of age. Animals treated for this experiment included: 9 WT male rats $(264.0 \mathrm{~g} \pm 22.9 \mathrm{~g}) ; 9 \mathrm{Cy} /+$ male rats $(251.1 \mathrm{~g} \pm 21.6) ; 6 \mathrm{WT}$ female rats $(202.8 \mathrm{~g} \pm 17.4 \mathrm{~g})$; and $7 \mathrm{Cy} /+$ female rats $(188.9 \mathrm{~g} \pm 14.3 \mathrm{~g})$, over 4 experiments. Untreated animals included: 4 WT male rats ( $274.3 \mathrm{~g} \pm 16 \mathrm{~g}) ; 5$ $\mathrm{Cy} /+$ male rats $(267.3 \mathrm{~g} \pm 16.6 \mathrm{~g}) ; 5 \mathrm{WT}$ female rats $(192 \mathrm{~g} \pm 12.1 \mathrm{~g}) ;$ and 5 $\mathrm{Cy} /+$ female rats $(193.8 \mathrm{~g} \pm 13.4 \mathrm{~g})$.

Citrate treatment of Han:SPRD rats began at week 7 until week 8 of age. As previously described (29), rats were given ad libitum access to drinking water supplemented with $55 \mathrm{mM}$ tripotassium citrate and $67 \mathrm{mM}$ citric acid. Kidneys were cut into sequential, $5-\mu \mathrm{m}$ sections and subjected to Pizzolato staining prior to manual quantification. Calcium deposits were identified as black aggregates within lumens. The analyst was blinded to the treatment of the animals. Animals treated for this experiment included the following: $3 \mathrm{WT}$ male rats $(244.5 \mathrm{~g} \pm 45.23$ g); $5 \mathrm{Cy} /+$ male rats $(249.8 .1 \mathrm{~g} \pm 21.6 \mathrm{~g}) ; 9 \mathrm{WT}$ female rats $(198.3 \mathrm{~g} \pm 16.8$ $\mathrm{g})$; and $3 \mathrm{Cy} /+$ female rats $(210.4 \mathrm{~g} \pm 11.5 \mathrm{~g})$, over 3 experiments.

PCK rat experiments were conducted at the Mayo Clinic College of Medicine (Supplemental Table 1) (46). From 3 to 10 weeks of age, 10 male and female WT and cystic rats were fed identical diets except for phosphorus content, which was $0.17 \%$ (TestDiet, 5857), $0.057 \%$ phosphorus (TestDiet, 5755), or 1.21\% phosphorus (TestDiet, 585Y).
Histological and immunohistological analyses. Birefringent $\mathrm{CaOx}$ crystals were detected in H\&E-stained paraffin-embedded sections by polarized light microscopy or Pizzolato's staining. CaP deposits were detected by von Kossa staining.

For immunofluorescence analysis, paraffin-embedded renal sections were rehydrated through a xylene and decreasing ethanol series, washed in Tris-buffered saline, and boiled in $10 \mathrm{mM}$ sodium citrate buffer (pH 6) for 5 minutes in a pressure cooker. The sections were cooled and then washed twice in TBST and blocked (1\% BSA, 0.1\% fish skin gelatin, $0.05 \%$ sodium azide in TBST) at $37^{\circ} \mathrm{C}$ for 30 minutes. Sections were incubated with the following antibodies diluted in blocking buffer overnight at $4^{\circ} \mathrm{C}$ : anti-p-S6 (Ser235/236) (Cell Signaling Technology, 4858); anti-pSTAT3 (Tyr705) (Cell Signaling Technology, 9145); anti-AQP1 (MilliporeSigma, AB2219); anti-AQP2 (Santa Cruz Biotechnology, sc-9880); anti-CALB1 (MilliporeSigma, C9848); anti-THP (Santa Cruz Biotechnology, sc020631); and anti-Ki67 (BD Pharmingen, 550609). Sections were incubated with $1 \%$ Sudan black diluted in $70 \%$ ethanol for 20 minutes and then washed twice in TBST for 5 minutes each wash. Sections were then incubated with fluorescence-labeled antibodies diluted in blocking buffer at $37^{\circ} \mathrm{C}$ for 1 hour (anti-rabbit DyLight 488 and 594, Thermo Fisher Scientific, 35552, and 35560; anti-mouse DyLight 488, Thermo Fisher Scientific, 35502) and/or fluorescence-labeled lectins LTL (Vector Laboratories, FL-1321) and DBA (Vector Laboratories, RL-1032). After washing, sections were postfixed in 10\% neutral-buffered formalin for 10 minutes, washed in TBS, and then mounted with ProLong Gold Antifade Mounting Medium with DAPI (Thermo Fisher Scientific).

For immunohistochemical analysis, paraffin-embedded sections were rehydrated as described above, followed by proteinase $\mathrm{K}(0.6 \mathrm{U} /$ $\mathrm{ml}$ in Tris plus EDTA [TE] buffer, $\mathrm{pH} 8$ ) digestion, treatment with 3\% $\mathrm{H}_{2} \mathrm{O}_{2}$, and blocking in $10 \%$ donkey serum. After successive incubations with a primary antibody (anti-CD68/SR-D10 [ED1], Novus Biologicals, NB600-985), a secondary antibody (anti-mouse biotin, Jackson ImmunoResearch, 715-065-151), and HRP conjugated streptavidin (streptavidin-HRP, BD Pharmingen, 554066), slides were developed using DAB peroxidase substrate (Vector SK-4100), followed by counterstaining with hematoxylin.

The cystic index was determined using H\&E-stained whole kidney sections, and analysis was conducted using ImageJ software (NIH) to determine the percentage of cystic area relative to the total kidney area. Manual determination of inclusive areas was used to exclude artifacts from tissue sectioning.

To measure the intrarenal location and size distribution of $\mathrm{CaOx}$ crystals and aggregates, ImageJ software was used on polarized light microscopic images of H\&E-stained whole kidney sections. Seven to ten fields from $\times 200$-magnified images were analyzed for both cortical and medullary areas.

ImageJ software was used for analysis of cell heights, tubule diameters, and lumen diameters of H\&E-stained whole kidney sections. Tubule diameters were measured from the basal membrane across the narrowest segment of a tubule to the basal membrane of the opposite side. Lumen diameters were determined by measuring the distance between the apical membranes across the narrowest portion of a tubule. Cell heights were determined by measuring cell height from the basal to apical membrane across a cell nucleus for 3 cells per tubule. The researcher performing the measurements was blinded to the treatments.

Immunoblotting. Kidneys were bisected and placed into a mortar cooled with liquid nitrogen, pulverized using a pestle, and stored at 
$-80^{\circ} \mathrm{C}$ for future analysis. Tissue samples were lysed in SDS sample buffer containing phosphatase and protease inhibitors (MilliporeSigma). Following standard SDS-PAGE and transfer, samples were analyzed using the following antibodies: anti-actin (Amersham Bioscience, N350); anti-p-S6 (Ser235/236) (Cell Signaling Technology, 4858); anti-S6 (Cell Signaling Technology, 2217); anti-SRC (Cell Signaling Technology, 2109); anti-p-SRC (Tyr416) (Cell Signaling Technology, 6943); anti-p-STAT3 (Tyr705) (Cell Signaling Technology, 9145); and anti-STAT3 (Cell Signaling Technology, 9139).

ADPKD patients and urine sample analyses. Urine samples from patients in the Emory ADPKD Cohort Study were collected and analyzed according to protocols approved by the IRBs of Emory University and the University of Alabama at Birmingham (UAB) (82). Oxalate levels were determined by ion chromatography mass spectrometry (IC-MS) (83) and citrate concentrations by liquid chromatography-MS (LC-MS).

Statistics. Data sets were tested for normality prior to a nonparametric, 1-tailed Mann-Whitney $U$ analysis with GraphPad Prism software after determining a non-normal distribution and $\chi^{2}$ analysis. Data on PCK rats were analyzed using a 2-way ANOVA and a 2-tailed, unpaired $t$ test. Box-and-whisker plots represent $90 \%$ of the values, with the median displayed as a line in between the second and third quartiles and the mean indicated by intersecting bars. A $P$ value of less than 0.05 was considered significant. Variances between statistically compared groups differed. Correlations between data on patients with ADPKD (urine citrate, creatinine, and albumin vs. TKV or EGFP) were analyzed using linear regression analysis and the sample Pearson's correlation coefficient.

Study approval. All animal experiments were conducted with approval of the IACUCs of the University of California Santa Barbara, the University of Florida, and the Mayo Clinic.

\section{Author contributions}

JAT and MR are co-first authors. This work was part of the PhD thesis by JAT, who made the initial significant discoveries and was assigned first position in the author list. JAT contributed to the design of the experiments, animal procedures, immunofluorescence, Western blotting, histological studies, statistical analysis, preparation of figures, and writing of the manuscript. MR contributed to histological, immunohistological, and statistical analyses and preparation of figures. LL conducted immunohistological analysis. CB contributed to animal procedures, immunoblotting, and tubule measurements. BH contributed biopsy specimens from patients with $\mathrm{PH} 1$ and provided clinical support. BDC provided Han:SPRD rats for experiments and experimental advice. VET, VS, and XW designed and conducted PCK rat experiments. SK contributed NPT2 $a^{-/-}$animals for analysis and contributed to experimental design. RPH conducted urine analyses and nephrolithiasis risk interpretation. MM designed and supervised the ADPKD patient data analyses. TW was the principal investigator and contributed to the design and supervision of most experiments, preparation of figures, and writing of the manuscript.

\section{Acknowledgments}

We thank Arlene Chapman (University of Chicago) for providing patients' urine samples. This work was supported by a grant from the NIH (DK109563) and gifts from the Lillian Goldman Charitable Trust and the Amy P. Goldman Foundation (to TW); a Jarrett Postdoctoral Fellowship from the Jarrett Family Fund for work in TW's laboratory; grants from the NIH (DK44863 and DK90728); the Mayo Clinic Robert M. and Billie Kelley Pirnie Translational PKD Research Center (to VET); a grant from the National Institute of Diabetes and Digestive and Kidney Diseases (NIDDK), NIH (to VET); an Early Postdoctoral Mobility Grant (P2BEP3-152098); and an Advanced Postdoctoral Mobility Grant (P300PB_167797) from the Swiss National Science Foundation (to MR). MM was supported in part by grants from the NIH (UAB Hepato/Renal Fibrocystic Disease Core Center, P30 DK074038 and DK097423) and the Office of Research and Development, Medical Research Service, Department of Veterans Affairs (1-I01-BX002298). Additional support was provided by the NIH-funded UAB-UCSD O'Brien Center (1P30 DK079337). The mass spectrometer used in these studies was obtained through a grant from the UAB Health Services Foundation General Endowment Fund.

Address correspondence to: Thomas Weimbs, Department of Molecular, Cellular and Developmental Biology, University of California Santa Barbara, Santa Barbara, California 93106-9625, USA. Phone: 805.893.4144; Email: weimbs@ucsb.edu.
1. Knight T, Schaefer C, Krasa H, Oberdhan D, Chapman A, Perrone RD. Medical resource utilization and costs associated with autosomal dominant polycystic kidney disease in the USA: a retrospective matched cohort analysis of private insurer data. Clinicoecon Outcomes Res. 2015;7:123-132.

2. Ong AC, Devuyst O, Knebelmann B, Walz G, ERA-EDTA Working Group for Inherited Kidney Diseases. Autosomal dominant polycystic kidney disease: the changing face of clinical management. Lancet. 2015;385(9981):1993-2002.

3. Erickson KF, Chertow GM, Goldhaber-Fiebert JD. Cost-effectiveness of tolvaptan in autosomal dominant polycystic kidney disease. Ann Intern Med. 2013;159(6):382-389.

4. Antignac $\mathrm{C}$, et al. The future of polycystic kidney disease research--As seen by the 12 Kaplan Awardees. J Am Soc Nephrol.
2015;26(9):2081-2095.

5. Lanktree MB, et al. Intrafamilial Variability of ADPKD. Kidney Int Rep. 2019;4(7):995-1003.

6. Weimbs T. Third-hit signaling in renal cyst formation. J Am Soc Nephrol. 2011;22(5):793-795.

7. Piontek K, Menezes LF, Garcia-Gonzalez MA, Huso DL, Germino GG. A critical developmental switch defines the kinetics of kidney cyst formation after loss of Pkd1. Nat Med. 2007;13(12):1490-1495.

8. Takakura A, et al. Renal injury is a third hit promoting rapid development of adult polycystic kidney disease. Hum Mol Genet. 2009;18(14):2523-2531.

9. Bastos AP, Piontek K, Silva AM, et al. Pkd1 haploinsufficiency increases renal damage and induces microcyst formation following ischemia/reperfusion. J Am Soc Nephrol. 2009;20(11):2389-2402.
10. Grantham JJ, et al. Detected renal cysts are tips of the iceberg in adults with ADPKD. Clin J Am Soc Nephrol. 2012;7(7):1087-1093.

11. Robertson WG. A method for measuring calcium crystalluria. Clin Chim Acta. 1969;26(1):105-110.

12. Khan SR, Kok DJ. Modulators of urinary stone formation. Front Biosci. 2004;9:1450-1482.

13. Fogazzi GB. Crystalluria: a neglected aspect of urinary sediment analysis. Nephrol Dial Transplant. 1996;11(2):379-387.

14. Khan SR. Calcium oxalate crystal interaction with renal tubular epithelium, mechanism of crystal adhesion and its impact on stone development. Urol Res. 1995;23(2):71-79.

15. Dimitrakov D, Simeonov S. Studies on nephrolithiasis in patients with autosomal dominant polycystic kidney disease. Folia Med (Plovdiv). 1994;36(3):27-30.

16. Torres VE, Wilson DM, Hattery RR, Segura JW. 
Renal stone disease in autosomal dominant polycystic kidney disease. Am J Kidney Dis. 1993;22(4):513-519.

17. Grampsas SA, et al. Anatomic and metabolic risk factors for nephrolithiasis in patients with autosomal dominant polycystic kidney disease. Am J Kidney Dis. 2000;36(1):53-57.

18. Nishiura JL, Neves RF, Eloi SR, Cintra SM, Ajzen SA, Heilberg IP. Evaluation of nephrolithiasis in autosomal dominant polycystic kidney disease patients. Clin JAm Soc Nephrol. 2009;4(4):838-844.

19. Mejías E, Navas J, Lluberes R, Martínez-Maldonado M. Hyperuricemia, gout, and autosomal dominant polycystic kidney disease. Am J Med Sci. 1989;297(3):145-148.

20. Panizo N, et al. Chronic kidney disease progression in patients with autosomal dominant polycystic kidney disease. Nefrologia. 2012;32(2):197-205.

21. Scales CD, et al. Changing gender prevalence of stone disease. JUrol. 2007;177(3):979-982.

22. Schrier RW, et al. Predictors of autosomal dominant polycystic kidney disease progression. JAm Soc Nephrol. 2014;25(11):2399-2418.

23. Khan SR, Glenton PA. Deposition of calcium phosphate and calcium oxalate crystals in the kidneys. JUrol. 1995;153(3 Pt 1):811-817.

24. Cowley BD, et al. Autosomal-dominant polycystic kidney disease in the rat. Kidney Int. 1993;43(3):522-534.

25. Nagao S, et al. Increased water intake decreases progression of polycystic kidney disease in the PCK rat. JAm Soc Nephrol. 2006;17(8):2220-2227.

26. Hopp K, Wang X, Ye H, Irazabal MV, Harris PC, Torres VE. Effects of hydration in rats and mice with polycystic kidney disease. Am J Physiol Renal Physiol. 2015;308(3):F261-F266.

27. Tanner GA, Tanner JA. Dietary citrate treatment of polycystic kidney disease in rats. Nephron Physiol. 2003;93(1):P14-P20.

28. Tanner GA, Tanner JA. Citrate therapy for polycystic kidney disease in rats. Kidney Int. 2000;58(5):1859-1869.

29. Tanner GA. Potassium citrate/citric acid intake improves renal function in rats with polycystic kidney disease. J Am Soc Nephrol. 1998;9(7):1242-1248.

30. Khan SR, Glenton PA. Calcium oxalate crysta deposition in kidneys of hypercalciuric mice with disrupted type IIa sodium-phosphate cotransporter. Am J Physiol Renal Physiol. 2008;294(5):F1109-F1115.

31. Talbot JJ, et al. Polycystin-1 regulates STAT activity by a dual mechanism. Proc Natl Acad Sci US A. 2011;108(19):7985-7990.

32. Shillingford JM, et al. The mTOR pathway is regulated by polycystin-1, and its inhibition reverses renal cystogenesis in polycystic kidney disease. Proc Natl Acad Sci US A. 2006;103(14):5466-5471.

33. Shillingford JM, Piontek KB, Germino GG, Weimbs T. Rapamycin ameliorates PKD resulting from conditional inactivation of Pkd1. J Am Soc Nephrol. 2010;21(3):489-497.

34. Shillingford JM, Leamon CP, Vlahov IR, Weimbs T. Folate-conjugated rapamycin slows progression of polycystic kidney disease. J Am Soc Nephrol. 2012;23(10):1674-1681.

35. Khan SR, Glenton PA. Experimental induction of calcium oxalate nephrolithiasis in mice. JUrol.
2010;184(3):1189-1196.

36. Khan SR, Finlayson B, Hackett RL. Experimental calcium oxalate nephrolithiasis in the rat. Role of the renal papilla. Am J Pathol. 1982;107(1):59-69.

37. Khan SR, Hackett RL. Retention of calcium oxalate crystals in renal tubules. Scanning Microsc. 1991;5(3):707-11; discussion 711.

38. Yang L, Besschetnova TY, Brooks CR, Shah JV, Bonventre JV. Epithelial cell cycle arrest in G2/M mediates kidney fibrosis after injury. Nat Med. 2010;16(5):535-43, 1p following 143.

39. Thomasova D, Anders HJ. Cell cycle control in the kidney. Nephrol Dial Transplant. 2015;30(10):1622-1630.

40. Cassini MF, et al. Mcp1 promotes macrophagedependent cyst expansion in autosomal dominant polycystic kidney disease. J Am Soc Nephrol. 2018;29(10):2471-2481.

41. Weimbs T. Are cyst-associated macrophages in polycystic kidney disease the equivalent to TAMs in cancer? J Am Soc Nephrol. 2018;29(10):2447-2448.

42. Anders HJ, et al. The macrophage phenotype and inflammasome component NLRP3 contributes to nephrocalcinosis-related chronic kidney disease independent from IL-1-mediated tissue injury. Kidney Int. 2018;93(3):656-669.

43. Breljak D, et al. In female rats, ethylene glycol treatment elevates protein expression of hepatic and renal oxalate transporter sat-1 (Slc26a1) without inducing hyperoxaluria. Croat Med J. 2015;56(5):447-459.

44. Yoshioka I, Tsujihata M, Momohara C, Akanae W, Nonomura N, Okuyama A. Effect of sex hormones on crystal formation in a stone-forming rat model. Urology. 2010;75(4):907-913.

45. McMartin K. Are calcium oxalate crystals involved in the mechanism of acute renal failure in ethylene glycol poisoning? Clin Toxicol (Phila). 2009;47(9):859-869.

46. Ward CJ, et al. The gene mutated in autosomal recessive polycystic kidney disease encodes a large, receptor-like protein. Nat Genet. 2002;30(3):259-269.

47. Renken C, Fischer DC, Kundt G, Gretz N, Haffner D. Inhibition of $\mathrm{mTOR}$ with sirolimus does not attenuate progression of liver and kidney disease in PCK rats. Nephrol Dial Transplant. 2011;26(1):92-100.

48. Ren XS, et al. Activation of the PI3K/mTOR pathway is involved in cystic proliferation of cholangiocytes of the PCK rat. PLOS ONE. 2014;9(1):e87660.

49. Sweeney WE, von Vigier RO, Frost P, Avner ED. Src inhibition ameliorates polycystic kidney disease. J Am Soc Nephrol. 2008;19(7):1331-1341.

50. Talbot JJ, et al. The cleaved cytoplasmic tail of polycystin-1 regulates Src-dependent STAT3 activation. J Am Soc Nephrol. 2014;25(8):1737-1748

51. Otnes B. Sex differences in the crystalline composition of stones from the upper urinary tract. Scand J Urol Nephrol. 1980;14(1):51-56.

52. Taylor EN, Curhan GC. Role of nutrition in the formation of calcium-containing kidney stones. Nephron Physiol. 2004;98(2):p55-p63.

53. Siener R. Impact of dietary habits on stone incidence. Urol Res. 2006;34(2):131-133.

54. Cochat P, Rumsby G. Primary hyperoxaluria.
N Engl J Med. 2013;369(7):649-658.

55. Ettinger B, Pak CY, Citron JT, Thomas C, AdamsHuet B, Vangessel A. Potassium-magnesium citrate is an effective prophylaxis against recurrent calcium oxalate nephrolithiasis. JUrol. 1997;158(6):2069-2073.

56. Torres VE, et al. Analysis of baseline parameters in the HALT polycystic kidney disease trials. Kidney Int. 2012;81(6):577-585.

57. Khan SR. Renal tubular damage/dysfunction: key to the formation of kidney stones. Urol Res. 2006;34(2):86-91.

58. Vervaet BA, Verhulst A, D'Haese PC, De Broe ME. Nephrocalcinosis: new insights into mechanisms and consequences. Nephrol Dial Transplant. 2009;24(7):2030-2035.

59. Evan AP, et al. Mechanism of formation of human calcium oxalate renal stones on Randall's plaque. Anat Rec (Hoboken). 2007;290(10):1315-1323.

60. Khan SR. Nephrocalcinosis in animal models with and without stones. Urol Res. 2010;38(6):429-438.

61. Khan SR, Finlayson B, Hackett RL. Histologic study of the early events in oxalate induced intranephronic calculosis. Invest Urol. 1979;17(3):199-202.

62. Worcester EM, et al. A test of the hypothesis that oxalate secretion produces proximal tubule crystallization in primary hyperoxaluria type I. Am J Physiol Renal Physiol. 2013;305(11):F1574-F1584.

63. Ermer T, Eckardt KU, Aronson PS, Knauf F. Oxalate, inflammasome, and progression of kidney disease. Curr Opin Nephrol Hypertens. 2016;25(4):363-371.

64. Cornu M, Albert V, Hall MN. mTOR in aging, metabolism, and cancer. Curr Opin Genet Dev. 2013;23(1):53-62.

65. Laplante M, Sabatini DM. mTOR signaling in growth control and disease. Cell. 2012;149(2):274-293.

66. Nishio S, et al. Pkd1 regulates immortalized proliferation of renal tubular epithelial cells through p53 induction and JNK activation. JClin Invest. 2005;115(4):910-918.

67. Dalagiorgou G, et al. Mechanosensor polycystin-1 potentiates differentiation of human osteoblastic cells by upregulating Runx 2 expression via induction of JAK2/STAT3 signaling axis. Cell Mol Life Sci. 2017;74(5):921-936.

68. Mulay SR, et al. Cytotoxicity of crystals involves RIPK3-MLKL-mediated necroptosis. Nat Commun. 2016;7:10274

69. Bell PD, et al. Loss of primary cilia upregulates renal hypertrophic signaling and promotes cystogenesis. J Am Soc Nephrol. 2011;22(5):839-848.

70. Brown JH, et al. Missense mutation in sterile alpha motif of novel protein SamCystin is associated with polycystic kidney disease in $(\mathrm{cy} /+)$ rat. JAm Soc Nephrol. 2005;16(12):3517-3526.

71. Nagao S, et al. Polycystic kidney disease in Han:SPRD Cy rats is associated with elevated expression and mislocalization of SamCystin. Am J Physiol Renal Physiol. 2010;299(5):F1078-F1086.

72. Stagner EE, Bouvrette DJ, Cheng J, Bryda EC. The polycystic kidney disease-related proteins Bicc1 and SamCystin interact. Biochem Biophys Res Commun. 2009;383(1):16-21.

73. Rothé B, et al. Bicc1 Polymerization Regulates the localization and silencing of bound mRNA. Mol 
Cell Biol. 2015;35(19):3339-3353.

74. Lian P, et al. Loss of polycystin-1 inhibits Bicc1 expression during mouse development. PLoS ONE. 2014;9(3):e88816.

75. Tran U, et al. The RNA-binding protein bicaudal $C$ regulates polycystin 2 in the kidney by antagonizing miR-17 activity. Development. 2010;137(7):1107-1116.

76. Wang S, et al. Fibrocystin/polyductin, found in the same protein complex with polycystin-2, regulates calcium responses in kidney epithelia. Mol Cell Biol. 2007;27(8):3241-3252.

77. Wang S, Wu M, Yao G, Zhang J, Zhou J. The cyto- plasmic tail of FPC antagonizes the full-length protein in the regulation of mTOR pathway. PLOS ONE. 2014;9(5):e95630.

78. Tanner JA, Tanner GA. Dietary potassium citrate does not harm the pcy mouse. Exp Biol Med (May wood). 2005;230(1):57-60.

79. Tanner GA, Vijayalakshmi K, Tanner JA. Effects of potassium citrate/citric acid intake in a mouse model of polycystic kidney disease. Nephron. 2000;84(3):270-273.

80. Ferraz RR, Fonseca JM, Germino GG, Onuchic LF, Heilberg IP. Determination of urinary lithogenic parameters in murine models orthologous to autosomal dominant polycystic kidney disease. Urolithiasis. 2014;42(4):301-307.

81. Thamilselvan S, Hackett RL, Khan SR. Lipid peroxidation in ethylene glycol induced hyperoxaluria and calcium oxalate nephrolithiasis. J Urol. 1997;157(3):1059-1063.

82. Zhou J, et al. Renal CD14 expression correlates with the progression of cystic kidney disease. Kidney Int. 2010;78(6):550-560.

83. Fargue S, Milliner DS, Knight J, Olson JB, Lowther WT, Holmes RP. Hydroxyproline metabolism and oxalate synthesis in primary hyperoxaluria. JAm Soc Nephrol. 2018;29(6):1615-1623. 